\title{
THE QUASI-KRONECKER FORM FOR MATRIX PENCILS*
}

\author{
THOMAS BERGER ${ }^{\dagger}$ AND STEPHAN TRENN ${ }^{\ddagger}$
}

\begin{abstract}
We study singular matrix pencils and show that the so-called Wong sequences yield a quasi-Kronecker form. This form decouples the matrix pencil into an underdetermined part, a regular part, and an overdetermined part. This decoupling is sufficient to fully characterize the solution behavior of the differential-algebraic equations associated with the matrix pencil. Furthermore, we show that the minimal indices of the pencil can be determined with only the Wong sequences and that the Kronecker canonical form is a simple corollary of our result; hence, in passing, we also provide a new proof for the Kronecker canonical form. The results are illustrated with an example given by a simple electrical circuit.
\end{abstract}

Key words. singular matrix pencil, Kronecker canonical form, differential algebraic equations

AMS subject classifications. 15A22, 15A21, 34A09, 34A30

DOI. $10.1137 / 110826278$

1. Introduction. We study (singular) linear matrix pencils

$$
s E-A \in \mathbb{K}^{m \times n}[s], \quad \text { where } \mathbb{K} \text { is } \mathbb{Q}, \mathbb{R}, \text { or } \mathbb{C},
$$

and the associated differential algebraic equation (DAE)

$$
E \dot{x}=A x+f,
$$

where $f$ is some inhomogeneity. In the context of DAEs it is natural to call matrix pencils $s E_{1}-A_{1}$ and $s E_{2}-A_{2}$ equivalent and write $s E_{1}-A_{1} \cong s E_{2}-A_{2}$ or $\left(E_{1}, A_{1}\right) \cong$ $\left(E_{2}, A_{2}\right)$, if there exist invertible matrices $S$ and $T$ such that

$$
S\left(s E_{1}-A_{2}\right) T=s E_{2}-A_{2} .
$$

In the literature this is also sometimes called strict or strong equivalence; see, e.g., [16, Chap. XII, section 1] and [21, Def. 2.1]. Based on this notion of equivalence it is of interest to find the "simplest" matrix pencil within an equivalence class. This problem was solved by Kronecker [19] (see also [16, 21]). Nevertheless, the analysis of matrix pencils is still an active research area (see, e.g., the recent paper [18]), mainly because of numerical issues, or to find ways to obtain the Kronecker canonical form efficiently (see, e.g., [36, 37, 8, 12, 13, 38]).

Our main goal in this paper is to highlight the importance of the Wong sequences [40] for the analysis of matrix pencils. The Wong sequences for the matrix pencil $s E-A$ are given by the following sequences of subspaces:

$$
\begin{array}{rlrl}
\mathcal{V}_{0} & :=\mathbb{K}^{n}, \quad \mathcal{V}_{i+1} & :=A^{-1}\left(E \mathcal{V}_{i}\right) \subseteq \mathbb{K}^{n} \\
\mathcal{W}_{0} & :=\{0\}, \quad \mathcal{W}_{i+1}:=E^{-1}\left(A \mathcal{W}_{i}\right) \subseteq \mathbb{K}^{n}
\end{array}
$$

*Received by the editors March 1, 2011; accepted for publication (in revised form) by C.-H. Guo February 7, 2012; published electronically May 3, 2012.

http://www.siam.org/journals/simax/33-2/82627.html

${ }^{\dagger}$ Institut für Mathematik, Technische Universität Ilmenau, Weimarer Straße 25, 98693 Ilmenau, Germany (thomas.berger@tu-ilmenau.de). This author's work was supported by DFG grant Il25/9.

${ }^{\ddagger}$ AG Technomathematik, Technische Universität Kaiserslautern, Erwin-Schrödinger-Str. Geb. 48, 67663 Kaiserslautern, Germany (trenn@mathematik.uni-kl.de). This author's work was supported by DFG grant Wi1458/10 while at the University of Würzburg. 
We will show (see Theorem 3.2 and Remark 3.3) that the Wong sequences are sufficient to completely characterize the solution behavior of the DAE (1.1) including the characterization of consistent initial values as well as constraints on the inhomogeneity $f$.

The Wong sequences can be traced back to Dieudonné [14]; however, his focus is only on the first of the two Wong sequences. Bernhard [10] and Armentano [3] used the Wong sequences to carry out a geometric analysis of matrix pencils. In [27] the first Wong sequence is introduced as a "fundamental geometric tool in the characterization of the subspace of consistent initial conditions" of a regular DAE. Both Wong sequences are introduced in [26] where the authors obtain a quasi-Kronecker staircase form; however, they did not consider both Wong sequences in combination so that the important role of the spaces $\mathcal{V}^{*} \cap \mathcal{W}^{*}, \mathcal{V}^{*}+\mathcal{W}^{*}, E \mathcal{V}^{*} \cap A \mathcal{W}^{*}, E \mathcal{V}^{*}+A \mathcal{W}^{*}$ (see Definition 2.1, Figure 2.1, and Theorem 2.3) is not highlighted. They also appear in $[1,2,20,35]$. In control theory modified versions of the Wong sequences (where $\operatorname{im} B$ is added to $E \mathcal{V}_{i}$ and $A \mathcal{W}_{i}$, resp.) have been studied extensively for not necessarily regular DAEs (see, e.g., [1, 4, 5, 6, 15, 22, 24, 28, 29]) and they have been found to be the appropriate tool to construct invariant subspaces, the reachability space, and provide a Kalman decomposition, just to name a few features. However, it seems that their relevance for a complete solution theory of DAEs (1.1) associated to a singular matrix pencil has been overlooked. We therefore believe that our solvability characterizations solely in terms of the Wong sequences are new.

The Wong sequences directly lead to a quasi-Kronecker triangular form (QKTF), i.e.,

$$
s E-A \cong\left[\begin{array}{ccc}
s E_{P}-A_{P} & * & * \\
0 & s E_{R}-A_{R} & * \\
0 & 0 & s E_{Q}-A_{Q}
\end{array}\right],
$$

where $s E_{R}-A_{R}$ is a regular matrix pencil. $s E_{P}-A_{P}$ is the "underdetermined" pencil and $s E_{Q}-A_{Q}$ is the "overdetermined" pencil (Theorem 2.3). With only a little more effort (invoking solvability of generalized Sylvester equations) we can get rid of the off-diagonal blocks and obtain a quasi-Kronecker form (QKF) (Theorem 2.6). From the latter it is easy to obtain the Kronecker canonical form (KCF) (Corollary 2.8) and hence another contribution of our work is a new proof for the KCF. We have to admit that our proof does not reach the elegance of the proof of Gantmacher [16]; however, Gantmacher does not provide any geometrical insight. At the other end of the spectrum, Armentano [3] uses the Wong sequences to obtain a result similar to ours (the QKTF but with more structured diagonal block entries); however, his approach is purely geometrical so that it is not directly possible to deduce the transformation matrices which are necessary to obtain the QKTF or QKF. Our result overcomes this disadvantage because it presents geometrical insights and, at the same time, is constructive. Furthermore, our work is self-contained in the sense that only results on regular matrix pencils are assumed to be well known.

Different from other authors, we do not primarily aim to decouple the regular part of the matrix pencil, because (1) the decoupling into three parts which have the solution properties "existence, but nonuniquess" (underdetermined part), "existence and uniqueness" (regular part), and "uniqueness, but possible nonexistence" (overdetermined part) seems very natural, and (2) the regular part can be further decoupled if necessary - again with the help of the Wong sequences as we showed in [9]. Furthermore, we are also not aiming for a staircase form as is common in the 
numerical literature; here the matrix pencils $s E_{P}-A_{P}, s E_{R}-A_{R}$ and $s E_{Q}-A_{Q}$ have no further structure, which has the advantage that the transformation matrices can be chosen to be simple (in any specified way).

Another advantage of our approach is that we respect the domain of the entries in the matrix pencil, e.g., if our matrices are real-valued, then all transformations remain real-valued. This is not true for results about the $\mathrm{KCF}$, because, due to possible complex eigenvalues and eigenvectors, even in the case of real-valued matrices it is necessary to allow for complex transformations and complex canonical forms. This is often undesirable, because if one starts with a real-valued matrix pencil, one would like to get real-valued results. Therefore, we formulated our results in such a way that they are valid for $\mathbb{K}=\mathbb{Q}, \mathbb{K}=\mathbb{R}$, and $\mathbb{K}=\mathbb{C}$. Especially for $\mathbb{K}=\mathbb{Q}$ it was also necessary to recheck known results, whether or not their proofs are also valid in $\mathbb{Q}$. We believe that the case $\mathbb{K}=\mathbb{Q}$ is of special importance because this allows the implementation of our approach in exact arithmetic which might be feasible if the matrices are sparse and not too big. In fact, we believe that the construction of the $\mathrm{QK}(\mathrm{T}) \mathrm{F}$ is also possible if the matrix pencil $s E-A$ contains symbolic entries as is common for the analysis of electrical circuits, where one might just add the symbol $R$ into the matrix instead of a specific value of the corresponding resistor. However, we have not formalized this, but our running example will show that it is no problem to keep symbolic entries in the matrix. This is a major difference of our approach compared to those available in literature which often aim for unitary transformation matrices (due to numerical stability) and are therefore not suitable for symbolic calculations.

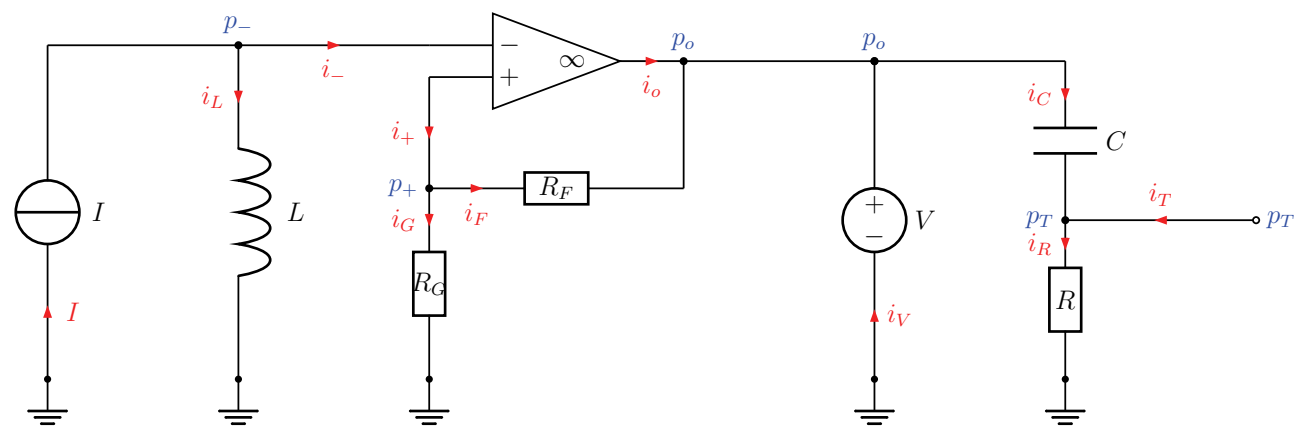

FIG. 1.1. An electrical circuit with sources and an open terminal used as the origin of the DAE (1.2) (used as a running example).

As a running example we use a DAE arising from an electrical circuit as shown in Figure 1.1. The electrical circuit has no practical purpose and is for academic analysis only. We assume that all the quantities $L, C, R, R_{G}, R_{F}$ are positive. To obtain the DAE description, let the state variable be given by $x=\left(p_{+}, p_{-}, p_{o}, p_{T}, i_{L}, i_{p}, i_{m}, i_{G}, i_{F}\right.$, $\left.i_{R}, i_{o}, i_{V}, i_{C}, i_{T}\right)^{\top}$ consisting of the node potentials and the currents through the branches. The inhomogeneity is $f=B u$ with $u=(I, V)^{\top}$ given by the sources and the matrix $B$ as below. The defining property of an ideal operational amplifier in feedback configuration is given by

$$
p_{+}=p_{-} \quad \text { and } \quad i_{+}=0=i_{-} .
$$

Collecting all defining equations of the circuit we obtain 13 equations for 14 state 
variables, which can be written as a DAE as follows:

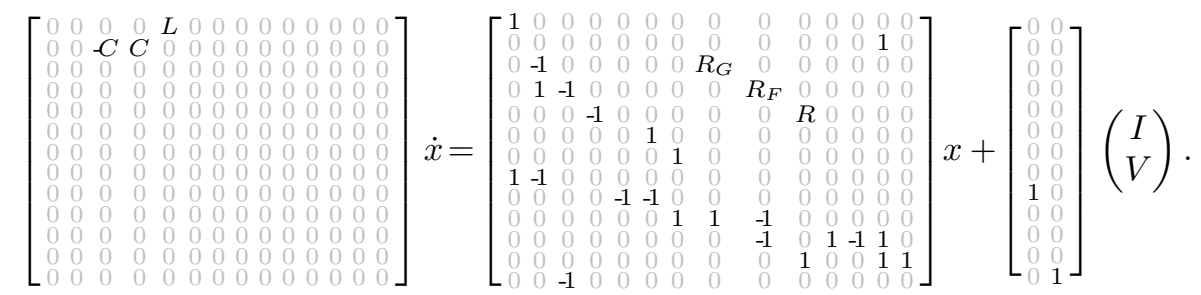

The coefficient matrices are not square, hence the corresponding matrix pencil $s E-A$ cannot be regular, and standard tools cannot be used to analyze this description of the circuit.

The paper is organized as follows. In section 2 we present our main results, in particular how the Wong sequences directly yield the QKTF (Theorem 2.3) and the minimal indices associated with the pencil (Theorem 2.9). Afterward, we show how the QKF can be used to fully characterize the solution behavior of the corresponding DAE in section 3. The proofs of the main results are carried out in section 5. Preliminary results are presented and proved in section 4 .

We close the introduction with the nomenclature used in this paper.

$$
\begin{array}{ll}
\mathbb{N} & \text { set of natural numbers with zero, } \mathbb{N}=\{0,1,2, \ldots\} \\
\mathbb{Q}, \mathbb{R}, \mathbb{C} & \text { field of rational, real, and complex numbers, resp. } \\
\mathbb{K} & \text { either } \mathbb{Q}, \mathbb{R}, \text { or } \mathbb{C} \\
\mathbf{G l}_{n}(\mathbb{K}) & \text { the set of invertible } n \times n \text { matrices over } \mathbb{K} \\
\mathbb{K}[s] & \text { the ring of polynomials with coefficients in } \mathbb{K} \\
\mathbb{K}^{m \times n} & \text { the set of } m \times n \text { matrices with entries in } \mathbb{K} \\
I \text { or } I_{n} & \text { the identity matrix of size } n \times n \text { for } n \in \mathbb{N} \\
A^{\top} & \text { the (conjugate) transpose of the matrix } A \in \mathbb{K}^{m \times n} \\
A \mathcal{S} & :=\left\{A x \in \mathbb{K}^{m} \mid x \in \mathcal{S}\right\}, \text { the image of } \mathcal{S} \subseteq \mathbb{K}^{n} \text { under } A \in \mathbb{K}^{m \times n} \\
A^{-1} \mathcal{S} & :=\left\{x \in \mathbb{K}^{n} \mid A x \in \mathcal{S}\right\}, \text { the preimage of } \mathcal{S} \subseteq \mathbb{K}^{m} \text { under } A \in \mathbb{K}^{m \times n} \\
A^{-\top} \mathcal{S} & :=\left(A^{\top}\right)^{-1} \mathcal{S} \\
\mathcal{S}^{\perp} & :=\left\{x \in \mathbb{K}^{n} \mid \forall s \in \mathcal{S}: x^{\top} s=0\right\}, \text { the orthogonal complement of } \mathcal{S} \subseteq \mathbb{K}^{n} \\
\operatorname{rank}_{\mathbb{C}}(\lambda E-A) & \text { the rank of }(\lambda E-A) \in \mathbb{C}^{m \times n}, E, A \in \mathbb{K}^{m \times n}, \text { for } \lambda \in \mathbb{C} ; \\
\mathcal{C}^{\infty} & \text { rank } \mathbb{C}_{\mathbb{C}}(\infty E-A):=\text { rank } E \\
\mathbb{D}_{\mathrm{pwC}} E & \text { the space of smooth }(\text { i.e., arbitrarily often differentiable) functions } \\
& \text { the space of piecewise-smooth distributions as introduced in }[33,34]
\end{array}
$$

2. Main results. As mentioned in the introduction, our approach is based on the Wong sequences which have been introduced in [40] for the analysis of matrix pencils. They can be calculated via a recursive subspace iteration. In a precursor [9] of this paper we used them to determine the quasi-Weierstraß form and it will turn out that they are the appropriate tool to determine a QKF as well.

Definition 2.1 (Wong sequences [40]). Consider a matrix pencil sE $-A \in$ $\mathbb{K}^{m \times n}[s]$. The Wong sequences corresponding to $s E-A$ are given by

$$
\begin{array}{rlrl}
\mathcal{V}_{0} & :=\mathbb{K}^{n}, & \mathcal{V}_{i+1} & :=A^{-1}\left(E \mathcal{V}_{i}\right) \subseteq \mathbb{K}^{n}, \\
\mathcal{W}_{0} & :=\{0\}, \quad \mathcal{W}_{i+1}:=E^{-1}\left(A \mathcal{W}_{i}\right) \subseteq \mathbb{K}^{n}
\end{array}
$$


Let $\mathcal{V}^{*}:=\bigcap_{i \in \mathbb{N}} \mathcal{V}_{i}$ and $\mathcal{W}^{*}:=\bigcup_{i \in \mathbb{N}} \mathcal{W}_{i}$ be the limits of the Wong sequences.

It is easy to see that the Wong sequences are nested, terminate, and satisfy

$$
\left.\begin{array}{l}
\exists k^{*} \in \mathbb{N} \forall j \in \mathbb{N}: \mathcal{V}_{0} \supsetneq \mathcal{V}_{1} \supsetneq \cdots \supsetneq \mathcal{V}_{k^{*}}=\mathcal{V}_{k^{*}+j}=\mathcal{V}^{*}=A^{-1}\left(E \mathcal{V}^{*}\right) \supseteq \operatorname{ker} A \\
\exists \ell^{*} \in \mathbb{N} \forall j \in \mathbb{N}: \mathcal{W}_{0} \subseteq \operatorname{ker} E=\mathcal{W}_{1} \subsetneq \cdots \subsetneq \mathcal{W}_{\ell^{*}}=\mathcal{W}_{\ell^{*}+j}=\mathcal{W}^{*}=E^{-1}\left(A \mathcal{W}^{*}\right)
\end{array}\right\}
$$

as well as

$$
A \mathcal{V}^{*} \subseteq E \mathcal{V}^{*} \quad \text { and } \quad E \mathcal{W}^{*} \subseteq A \mathcal{W}^{*}
$$

For our example DAE (1.2), we obtain

$$
\mathcal{V}_{1}=\operatorname{im}\left[\begin{array}{cccc}
0 & 0 & 0 \\
0 & 0 & 0 \\
0 & 0 & 0 \\
0 & 0 & 0 \\
0 & 0 & 0 \\
0 & 0 & 0 \\
0 & 0 & 0 \\
0 & 0 & 0 \\
0 & 0 & 0 \\
0 & 1 & 0 \\
0 & 0 & 1 \\
0 & -1 & 1 \\
-1 & 1 & -1
\end{array}\right]=\mathcal{V}^{*}
$$

and

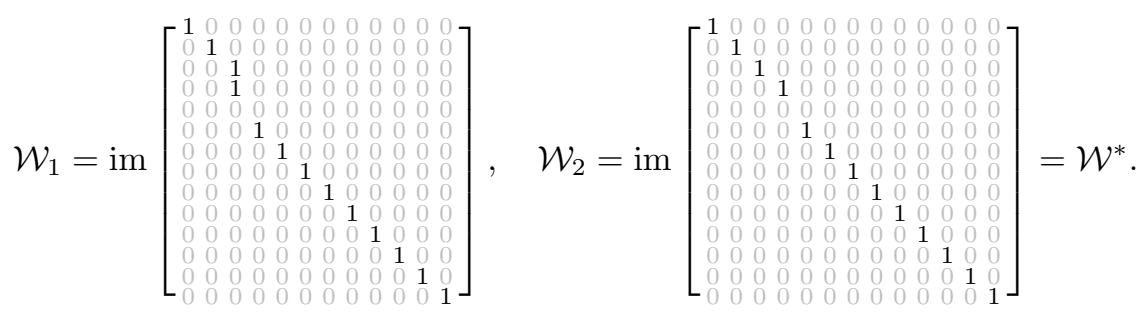

We carried out the calculation with MATLAB and its Symbolic Tool Box and the following short function for calculating the preimage:

LISTING 1

MATLAB function for calculating a basis of the preimage $A^{-1}(\mathrm{im} S)$ for some matrices $A$ and $S$.

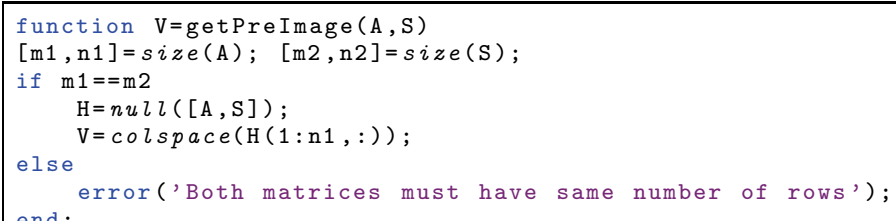

Before stating our main result we repeat the result concerning the Wong sequences and regular matrix pencils.

THEOREM 2.2 (the regular case, see [9]). Consider a regular matrix pencil sE$A \in \mathbb{K}^{m \times n}[s]$, i.e., $m=n$ and $\operatorname{det}(s E-A) \in \mathbb{K}[s] \backslash\{0\}$. Let $\mathcal{V}^{*}$ and $\mathcal{W}^{*}$ be the limits of the corresponding Wong sequences. Choose any full rank matrices $V$ and $W$ such that $\operatorname{im} V=\mathcal{V}^{*}$ and $\operatorname{im} W=\mathcal{W}^{*}$. Then $T=[V, W]$ and $S=[E V, A W]^{-1}$ are invertible and put the matrix pencil $s E-A$ into quasi-Weierstraß form,

$$
(S E T, S A T)=\left(\left[\begin{array}{cc}
I & 0 \\
0 & N
\end{array}\right],\left[\begin{array}{ll}
J & 0 \\
0 & I
\end{array}\right]\right)
$$


where $J \in \mathbb{K}^{n_{J} \times n_{J}}, n_{J} \in \mathbb{N}$, and $N \in \mathbb{K}^{n_{N} \times n_{N}}, n_{N}=n-n_{J}$, is a nilpotent matrix. In particular, when choosing $T_{J}$ and $T_{N}$ such that $T_{J}^{-1} J T_{J}$ and $T_{N}^{-1} N T_{N}$ are in Jordan canonical form, then $S^{\prime}=\left[E V T_{J}, A W T_{N}\right]^{-1}$ and $T^{\prime}=\left[V T_{J}, W T_{N}\right]$ put the regular matrix pencil $s E-A$ into Weierstraß canonical form.

Important consequences of Theorem 2.2 for the Wong sequences in the regular case are

$$
\begin{array}{rlrl}
\mathcal{V}^{*} \cap \mathcal{W}^{*} & =\{0\}, & E \mathcal{V}^{*} \cap A \mathcal{W}^{*}=\{0\}, \\
\mathcal{V}^{*}+\mathcal{W}^{*}=\mathbb{K}^{n}, & E \mathcal{V}^{*}+A \mathcal{W}^{*}=\mathbb{K}^{n} .
\end{array}
$$

These properties do not hold anymore for a general matrix pencil $s E-A$; see Figure 2.1 for an illustration of the situation.
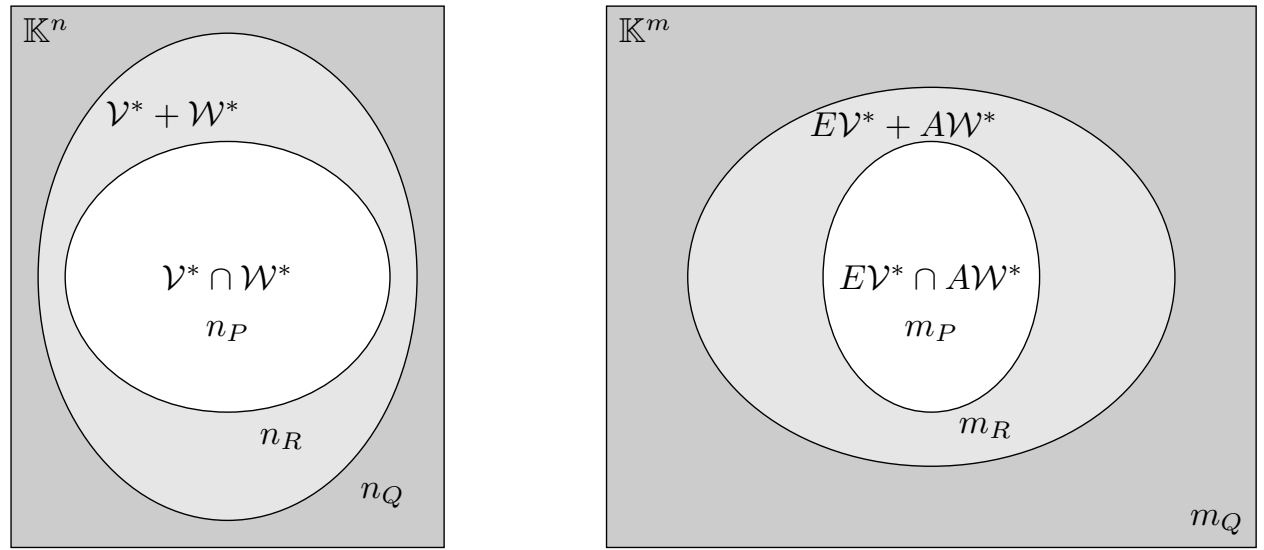

FIG. 2.1. The relationship of the limits $\mathcal{V}^{*}$ and $\mathcal{W}^{*}$ of the Wong sequences of the matrix pencil $s E-A \in \mathbb{K}^{m \times n}[s]$ in the general case; the numbers $n_{P}, n_{R}, n_{Q}, m_{P}, m_{R}, m_{Q} \in \mathbb{N}$ denote the (difference of the) dimensions of the corresponding spaces.

We are now ready to present our first main result which states that the knowledge of the spaces $\mathcal{V}^{*}$ and $\mathcal{W}^{*}$ is sufficient to obtain the $Q K T F$, which already captures most structural properties of the matrix pencil $s E-A$. With the help of the Wong sequences Armentano [3] already obtained a similar result; however, his aim was to obtain a triangular form where the diagonal blocks are in canonical form. Therefore, his result is more general than ours, however, the price is a more complicated proof and it is also not clear how to obtain the transformation matrices explicitly.

TheOREM 2.3 (quasi-Kronecker triangular form (QKTF)). Let $s E-A \in \mathbb{K}^{m \times n}[s]$ and consider the corresponding limits $\mathcal{V}^{*}$ and $\mathcal{W}^{*}$ of the Wong sequences as in Definition 2.1. Choose any full rank matrices $P_{1} \in \mathbb{K}^{n \times n_{P}}, P_{2} \in \mathbb{K}^{m \times m_{P}}, R_{1} \in \mathbb{K}^{n \times n_{R}}$, $R_{2} \in \mathbb{K}^{m \times m_{R}}, Q_{1} \in \mathbb{K}^{n \times n_{Q}}, Q_{2} \in \mathbb{K}^{m \times m_{Q}}$ such that

$$
\begin{aligned}
\operatorname{im} P_{1} & =\mathcal{V}^{*} \cap \mathcal{W}^{*}, & \operatorname{im} P_{2} & =E \mathcal{V}^{*} \cap A \mathcal{W}^{*}, \\
\mathcal{V}^{*} \cap \mathcal{W}^{*} \oplus \operatorname{im} R_{1} & =\mathcal{V}^{*}+\mathcal{W}^{*}, & E \mathcal{V}^{*} \cap A \mathcal{W}^{*} \oplus \operatorname{im} R_{2} & =E \mathcal{V}^{*}+A \mathcal{W}^{*}, \\
\left(\mathcal{V}^{*}+\mathcal{W}^{*}\right) \oplus \operatorname{im} Q_{1} & =\mathbb{K}^{n}, & \left(E \mathcal{V}^{*}+A \mathcal{W}^{*}\right) \oplus \operatorname{im} Q_{2} & =\mathbb{K}^{m}
\end{aligned}
$$

Then $T_{\text {trian }}=\left[P_{1}, R_{1}, Q_{1}\right] \in \mathbf{G l}_{n}(\mathbb{K})$ and $S_{\text {trian }}=\left[P_{2}, R_{2}, Q_{2}\right]^{-1} \in \mathbf{G l}_{m}(\mathbb{K})$ transform $s E-A$ in $\mathrm{QKTF}$ :

$$
\left(S_{\text {trian }} E T_{\text {trian }}, S_{\text {trian }} A T_{\text {trian }}\right)=\left(\left[\begin{array}{ccc}
E_{P} & E_{P R} & E_{P Q} \\
0 & E_{R} & E_{R Q} \\
0 & 0 & E_{Q}
\end{array}\right],\left[\begin{array}{ccc}
A_{P} & A_{P R} & A_{P Q} \\
0 & A_{R} & A_{R Q} \\
0 & 0 & A_{Q}
\end{array}\right]\right)
$$


where

(i) $E_{P}, A_{P} \in \mathbb{K}^{m_{P} \times n_{P}}, m_{P}<n_{P}$, are such that $\operatorname{rank}_{\mathbb{C}}\left(\lambda E_{P}-A_{P}\right)=m_{P}$ for all $\lambda \in \mathbb{C} \cup\{\infty\}$,

(ii) $E_{R}, A_{R} \in \mathbb{K}^{m_{R} \times n_{R}}, m_{R}=n_{R}$, with $s E_{R}-A_{R}$ regular, i.e., $\operatorname{det}\left(s E_{R}-A_{R}\right) \not \equiv$ 0 ,

(iii) $E_{Q}, A_{Q} \in \mathbb{K}^{m_{Q} \times n_{Q}}, m_{Q}>n_{Q}$, are such that $\operatorname{rank}_{\mathbb{C}}\left(\lambda E_{Q}-A_{Q}\right)=n_{Q}$ for all $\lambda \in \mathbb{C} \cup\{\infty\}$.

The proof is carried out in section 5 .

Remark 2.4. The sizes of the blocks in (2.3) are uniquely given by the matrix pencil $s E-A$ because they depend only on the subspaces constructed by the Wong sequences and not on the choice of bases thereof. It is also possible that $m_{P}=0$ (or $n_{Q}=0$ ) which means that there are matrices with no rows (or no columns). On the other hand, if $n_{P}=0, n_{R}=0$, or $m_{Q}=0$, then the $P$-blocks, $R$-blocks, or $Q$-blocks are not present at all. Furthermore, it is easily seen that if $s E-A$ fulfills (i), (ii), or (iii) itself, then $s E-A$ is already in QKTF with $T_{\text {trian }}=P_{1}=I, T_{\text {trian }}=R_{1}=I$, or $T_{\text {trian }}=Q_{1}=I$, and $S_{\text {trian }}=P_{2}^{-1}=I, S_{\text {trian }}=R_{2}^{-1}=I$, or $S_{\text {trian }}=Q_{2}^{-1}=I$.

Remark 2.5. From Lemma 4.4 we know that $E\left(\mathcal{V}^{*} \cap \mathcal{W}^{*}\right)=E \mathcal{V}^{*} \cap A \mathcal{W}^{*}=$ $A\left(\mathcal{V}^{*} \cap \mathcal{W}^{*}\right)$; hence

$$
E \mathcal{V}^{*} \cap A \mathcal{W}^{*}=E\left(\mathcal{V}^{*} \cap \mathcal{W}^{*}\right)+A\left(\mathcal{V}^{*} \cap \mathcal{W}^{*}\right)
$$

Furthermore, due to (2.2),

$$
E \mathcal{V}^{*}+A \mathcal{W}^{*}=E\left(\mathcal{V}^{*}+\mathcal{W}^{*}\right)+A\left(\mathcal{V}^{*}+\mathcal{W}^{*}\right)
$$

Hence the subspace pairs $\left(\mathcal{V}^{*} \cap \mathcal{W}^{*}, E \mathcal{V}^{*} \cap A \mathcal{W}^{*}\right)$ and $\left(\mathcal{V}^{*}+\mathcal{W}^{*}, E \mathcal{V}^{*}+A \mathcal{W}^{*}\right)$ are reducing subspaces of the matrix pencil $s E-A$ in the sense of [37] and are in fact the minimal and maximal reducing subspaces. ${ }^{1}$

In our example (1.2) we have

$$
\mathcal{V}^{*} \cap \mathcal{W}^{*}=\mathcal{V}^{*}, \mathcal{V}^{*}+\mathcal{W}^{*}=\mathcal{W}^{*}
$$

and, with $K:=\frac{R_{G}+R_{F}}{R_{G}}$,

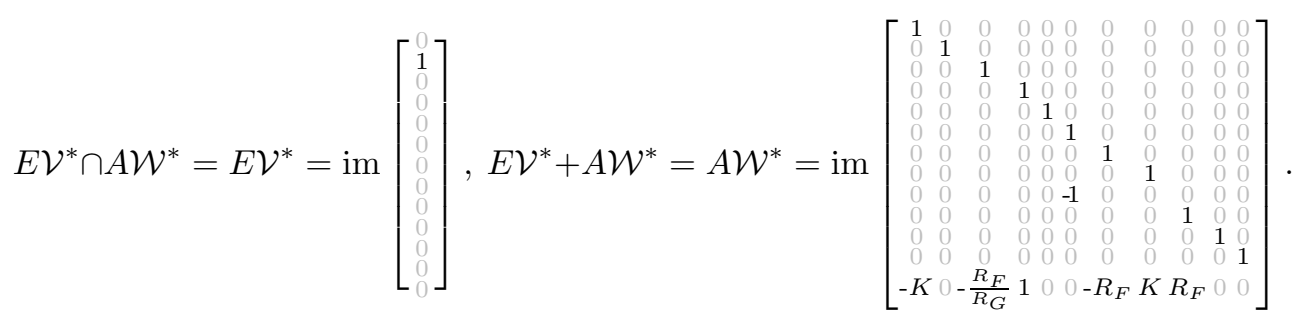

Therefore, we can choose

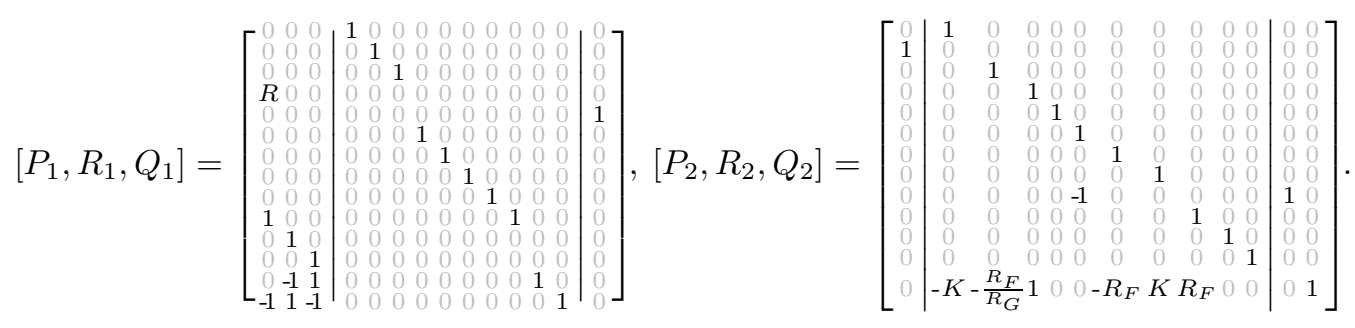

\footnotetext{
${ }^{1}$ We thank the anonymous reviewer for making us aware of this relationship.
} 
With this choice we obtain the following QKTF for our example:

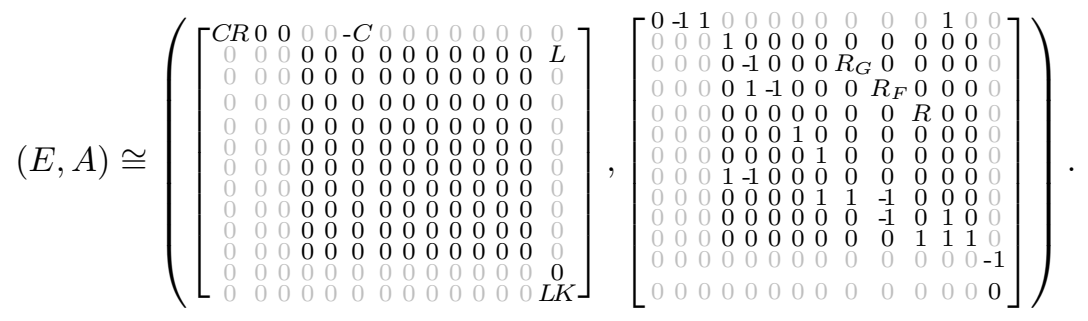

The QKTF is already useful for the analysis of the matrix pencil $s E-A$ and the associated DAE $E \dot{x}=A x+f$. However, a complete decoupling of the different parts, i.e., a block triangular form, is more satisfying from a theoretical viewpoint and is also a necessary step to obtaining the $\mathrm{KCF}$ as a corollary. In the next result we show that we can transform any matrix pencil $s E-A$ into a block triangular form, which we call quasi-Kronecker form ( $Q K F$ ) because all the important features of the $\mathrm{KCF}$ are captured. In fact, it turns out that the diagonal blocks of the QKTF (2.3) already are the diagonal blocks of the QKF.

THEOREM 2.6 (QKF). Using the notation from Theorem 2.3 the following equations are solvable for matrices $F_{1}, F_{2}, G_{1}, G_{2}, H_{1}, H_{2}$ of appropriate size:

$$
\begin{aligned}
& 0=E_{R Q}+E_{R} F_{1}+F_{2} E_{Q}, \\
& 0=A_{R Q}+A_{R} F_{1}+F_{2} A_{Q}, \\
& 0=E_{P R}+E_{P} G_{1}+G_{2} E_{R}, \\
& 0=A_{P R}+A_{P} G_{1}+G_{2} A_{R}, \\
& 0=\left(E_{P Q}+E_{P R} F_{1}\right)+E_{P} H_{1}+H_{2} E_{Q}, \\
& 0=\left(A_{P Q}+A_{P R} F_{1}\right)+A_{P} H_{1}+H_{2} A_{Q},
\end{aligned}
$$

and for any such matrices let

$$
S:=\left[\begin{array}{ccc}
I & -G_{2} & -H_{2} \\
0 & I & -F_{2} \\
0 & 0 & I
\end{array}\right]^{-1} S_{\text {trian }}=\left[P_{2}, R_{2}-P_{2} G_{2}, Q_{2}-P_{2} H_{2}-R_{2} F_{2}\right]^{-1}
$$

and

$$
T:=T_{\text {trian }}\left[\begin{array}{ccc}
I & G_{1} & H_{1} \\
0 & I & F_{1} \\
0 & 0 & I
\end{array}\right]=\left[P_{1}, R_{1}+P_{1} G_{1}, Q_{1}+P_{1} H_{1}+R_{1} F_{1}\right] .
$$

Then $S \in \mathbf{G l}_{m}(\mathbb{K})$ and $T \in \mathbf{G l}_{n}(\mathbb{K})$ put $s E-A$ in $\mathrm{QKF}$

$$
(S E T, S A T)=\left(\left[\begin{array}{ccc}
E_{P} & 0 & 0 \\
0 & E_{R} & 0 \\
0 & 0 & E_{Q}
\end{array}\right],\left[\begin{array}{ccc}
A_{P} & 0 & 0 \\
0 & A_{R} & 0 \\
0 & 0 & A_{Q}
\end{array}\right]\right),
$$

where the block diagonal entries are the same as for the QKTF (2.3). In particular, the QKF (without the transformation matrices $S$ and $T$ ) can be obtained with only the Wong sequences (i.e., without solving (2.4)). Furthermore, the QKF (2.5) is unique in the following sense:

$$
\begin{aligned}
& (E, A) \cong\left(E^{\prime}, A^{\prime}\right) \\
& \quad \Leftrightarrow\left(E_{P}, A_{P}\right) \cong\left(E_{P}^{\prime}, A_{P}^{\prime}\right),\left(E_{R}, A_{R}\right) \cong\left(E_{R}^{\prime}, A_{R}^{\prime}\right),\left(E_{Q}, A_{Q}\right) \cong\left(E_{Q}^{\prime}, A_{Q}^{\prime}\right),
\end{aligned}
$$


where $E_{P}^{\prime}, A_{P}^{\prime}, E_{R}^{\prime}, A_{R}^{\prime}, E_{P}^{\prime}, A_{P}^{\prime}$ are the corresponding blocks of the $Q K F$ of the matrix pencil $s E^{\prime}-A^{\prime}$.

The proof is carried out in section 5. In order to actually find solutions of (2.4), the following remark might be helpful.

Remark 2.7. Matrix equations of the form

$$
\begin{aligned}
& 0=M+P X+Y Q, \\
& 0=R+S X+Y T
\end{aligned}
$$

for given matrices $M, P, Q, R, S, T$ of appropriate size can be written equivalently as a standard linear system

$$
\left[\begin{array}{cc}
I \otimes P & Q^{\top} \otimes I \\
I \otimes S & T^{\top} \otimes I
\end{array}\right]\left(\begin{array}{c}
\operatorname{vec}(X) \\
\operatorname{vec}(Y)
\end{array}\right)=-\left(\begin{array}{c}
\operatorname{vec}(M) \\
\operatorname{vec}(R)
\end{array}\right)
$$

where $\otimes$ denotes the Kronecker product of matrices and vec $(H)$ denotes the vectorization of the matrix $H$ obtained by stacking all columns of $H$ over each other.

For our example (1.2) we already know the QKF, because, as mentioned in Theorem 2.6, the diagonal blocks are the same as for the QKTF. However, we do not yet know the final transformation matrices which yield the QKF. Therefore, we have to find solutions of (2.4):

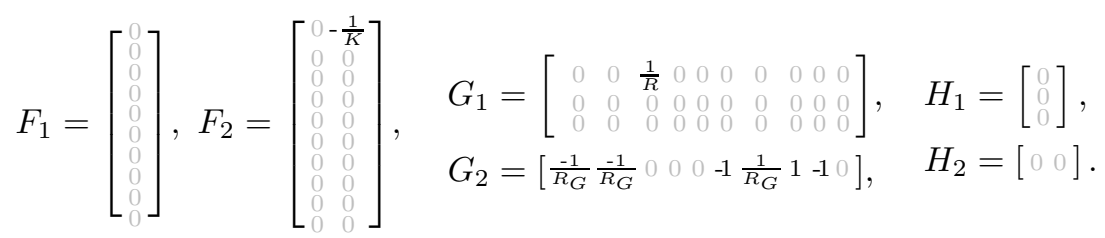

The transformation matrices $S$ and $T$ which put our example into a QKF are then

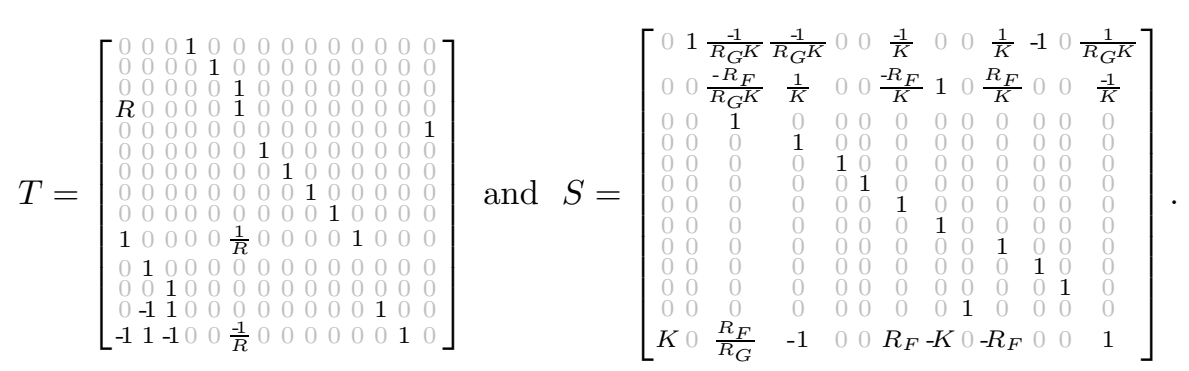

Finally, an analysis of the matrix pencils $s E_{P}-A_{P}$ and $s E_{Q}-A_{Q}$ in (2.5), invoking Lemma 4.12 and Corollary 4.13, together with Theorem 2.2, now allows us to obtain the KCF as a corollary.

Corollary 2.8. For every matrix pencil $s E-A \in \mathbb{K}^{m \times n}[s]$ there exist transformation matrices $S \in \mathbf{G l}_{m}(\mathbb{C})$ and $T \in \mathbf{G l}_{n}(\mathbb{C})$ such that, for $a, b, c, d \in \mathbb{N}$ and $\varepsilon_{1}, \ldots, \varepsilon_{a}, \rho_{1}, \ldots, \rho_{b}, \sigma_{1}, \ldots, \sigma_{c}, \eta_{1}, \ldots, \eta_{d} \in \mathbb{N}, S(s E-A) T=$

$$
\operatorname{diag}\left(\mathcal{P}_{\varepsilon_{1}}(s), \ldots, \mathcal{P}_{\varepsilon_{a}}(s), \mathcal{J}_{\rho_{1}}(s), \ldots, \mathcal{J}_{\rho_{b}}(s), \mathcal{N}_{\sigma_{1}}(s), \ldots, \mathcal{N}_{\sigma_{c}}(s), \mathcal{Q}_{\eta_{1}}(s), \ldots, \mathcal{Q}_{\eta_{d}}(s)\right)
$$


where

$$
\begin{aligned}
& \mathcal{P}_{\varepsilon}(s)=s\left[\begin{array}{cccc}
0 & 1 & & \\
& \ddots & \ddots & \\
& & 0 & 1
\end{array}\right]-\left[\begin{array}{cccc}
1 & 0 & & \\
& \ddots & \ddots & \\
& & 1 & 0
\end{array}\right] \in \mathbb{K}^{\varepsilon \times(\varepsilon+1)}[s], \varepsilon \in \mathbb{N}, \\
& \mathcal{J}_{\rho}(s)=s I-\left[\begin{array}{cccc}
\lambda & 1 & & \\
& \ddots & \ddots & \\
& & \ddots & 1 \\
& & & \lambda
\end{array}\right] \in \mathbb{C}^{\rho \times \rho}[s], \rho \in \mathbb{N}, \lambda \in \mathbb{C}, \\
& \mathcal{N}_{\sigma}(s)=s\left[\begin{array}{cccc}
0 & 1 & & \\
& \ddots & \ddots & \\
& & \ddots & 1 \\
& & & 0
\end{array}\right]-I \in \mathbb{K}^{\sigma \times \sigma}[s], \sigma \in \mathbb{N} \\
& \mathcal{Q}_{\eta}(s)=s\left[\begin{array}{ccc}
0 & & \\
1 & \ddots & \\
& \ddots & 0 \\
& & 1
\end{array}\right]-\left[\begin{array}{lll}
1 & & \\
0 & \ddots & \\
& \ddots & 1 \\
& & 0
\end{array}\right] \in \mathbb{K}^{(\eta+1) \times \eta}[s], \eta \in \mathbb{N} .
\end{aligned}
$$

The numbers $\rho_{i}, \sigma_{i}, \varepsilon_{i}$, and $\eta_{i}$ in Corollary 2.8 are usually called (degrees of) elementary divisors and minimal indices and play an important role in the analysis of matrix pencils; see, e.g., $[16,23,24,25]$. More precisely, $\rho_{1}, \ldots, \rho_{b}$ are the degrees of the finite elementary divisors, $\sigma_{1}, \ldots, \sigma_{c}$ are the degrees of the infinite elementary divisors, $\varepsilon_{1}, \ldots, \varepsilon_{a}$ are the columns minimal indices, and $\eta_{1}, \ldots, \eta_{d}$ are the row minimal indices. The minimal indices completely determine (under the standing assumption that $0 \leq \varepsilon_{1} \leq \cdots \leq \varepsilon_{a}$ and $0 \leq \eta_{1} \leq \cdots \leq \eta_{d}$ ) the singular part of the KCF. The following result shows that the minimal indices can actually be determined via the Wong sequences. Another method for determining the minimal indices via the control theoretic version of the Wong sequences can be found in [24, Prop. 2.2]; however, the minimal indices there correspond to a feedback canonical form.

Theorem 2.9 (minimal indices). Consider the Wong sequences (2.1) and the notation of Corollary 2.8. Let $\mathcal{K}=\mathcal{V}^{*} \cap \mathcal{W}^{*}, \widehat{\mathcal{W}}_{i}:=\left(E \mathcal{V}_{i-1}\right)^{\perp}, i=1,2, \ldots$, and $\widehat{\mathcal{K}}=\left(A \mathcal{W}^{*}\right)^{\perp} \cap\left(E \mathcal{V}^{*}\right)^{\perp}$. Then

$$
a=\operatorname{dim}\left(\mathcal{W}_{1} \cap \mathcal{K}\right), \quad d=\operatorname{dim}\left(\widehat{\mathcal{W}}_{1} \cap \widehat{\mathcal{K}}\right),
$$

and with, for $i=0,1,2, \ldots$,

$$
\Delta_{i}:=\operatorname{dim}\left(\mathcal{W}_{i+2} \cap \mathcal{K}\right)-\operatorname{dim}\left(\mathcal{W}_{i+1} \cap \mathcal{K}\right), \quad \widehat{\Delta}_{i}:=\operatorname{dim}\left(\widehat{\mathcal{W}}_{i+2} \cap \widehat{\mathcal{K}}\right)-\operatorname{dim}\left(\widehat{\mathcal{W}}_{i+1} \cap \widehat{\mathcal{K}}\right),
$$

it holds that

$$
\varepsilon_{1}=\cdots=\varepsilon_{a-\Delta_{0}}=0, \quad \varepsilon_{a-\Delta_{i-1}+1}=\cdots=\varepsilon_{a-\Delta_{i}}=i, \quad i=1,2, \ldots,
$$

and

$$
\eta_{1}=\cdots=\eta_{d-\widehat{\Delta}_{0}}=0, \quad \eta_{d-\widehat{\Delta}_{i-1}+1}=\cdots=\eta_{d-\widehat{\Delta}_{i}}=i, \quad i=1,2, \ldots
$$


Furthermore, the minimal indices are invariant under matrix pencil equivalence. In particular, the KCF is unique.

The proof is carried out in section 5 . This proof uses the KCF and, therefore, in particular it uses Lemma 4.12 and Corollary 4.13, which provide an explicit method to obtain the KCF for the full rank matrix pencils $s E_{P}-A_{P}$ and $s E_{Q}-A_{Q}$ in the QKF (2.5). However, if one is only interested in the singular part of the KCF (without the necessary transformation), the above result shows that knowledge of the Wong sequences is already sufficient; there is no need to actually carry out the tedious calculations of Lemma 4.12 and Corollary 4.13.

Note that $\Delta_{i-1}=\Delta_{i}$ or $\widehat{\Delta}_{i-1}=\widehat{\Delta}_{i}$ is possible for some $i$. In that case there are no minimal indices of value $i$ because the corresponding index range is empty. Furthermore, once $\Delta_{i}=0$ or $\widehat{\Delta}_{\widehat{i}}=0$ for some index $i$ or $\widehat{i}$, then $\Delta_{i+j}=0$ and $\widehat{\Delta}_{\widehat{i}+\widehat{j}}=0$ for all $j \geq 0$ and $\widehat{j} \geq 0$. In particular, there are no minimal indices with values larger than $i$ and $\hat{i}$, respectively.

3. Application of the QK(T)F to DAE solution theory. In this section we study the DAE (1.1)

$$
E \dot{x}=A x+f
$$

corresponding to the matrix pencil $s E-A \in \mathbb{R}^{m \times n}[s]$. Note that we restrict ourselves here to the field $\mathbb{K}=\mathbb{R}$, because (1) the vast majority of DAEs arising from modeling physical phenomena are not complex valued, (2) all the results for $\mathbb{K}=\mathbb{R}$ carry over to $\mathbb{K}=\mathbb{C}$ without modification (the converse is not true in general), (3) the case $\mathbb{K}=\mathbb{Q}$ is rather artificial when considering solutions of the DAE (1.1), because then we had to consider functions $f: \mathbb{R} \rightarrow \mathbb{Q}$ or even $f: \mathbb{Q} \rightarrow \mathbb{Q}$.

We first have to decide in which (function) space we actually consider the DAE (1.1). To avoid problems with differentiability, one suitable choice is the space of smooth functions $\mathcal{C}^{\infty}$, i.e., we consider smooth inhomogeneities $f \in\left(\mathcal{C}^{\infty}\right)^{m}$ and smooth $x \in\left(\mathcal{C}^{\infty}\right)^{n}$. Unfortunately, this excludes the possibility of considering step functions as inhomogeneities which occur rather frequently. It is well known that the solutions of DAEs might involve derivatives of the inhomogeneities, hence jumps in the inhomogeneity might lead to nonexistence of solutions due to a lack of differentiability. However, this is not a "structural nonexistence" since every smooth approximation of the jump could lead to well defined solutions. Therefore, one might extend the solution space by considering distributions (or generalized functions) as formally introduced by Schwartz [31]. The advantage of this larger solution space is that each distribution is "smooth," in particular the unit step function (Heaviside function) has a derivative: the Dirac impulse. Unfortunately, the whole space of distributions is too large; for example it is, in general, not possible to speak of an initial value, because evaluation of a distribution at a specific time is not defined. To overcome this obstacle we consider the smaller space of piecewise-smooth distributions $\mathbb{D}_{\mathrm{pw}} \infty$ as introduced in $[33,34]$. For piecewise-smooth distributions a left- and right-sided evaluation is possible, i.e., for $D \in \mathbb{D}_{\text {pwC }}$ the values $D(t-) \in \mathbb{R}$ and $D(t+) \in \mathbb{R}$ are well defined for all $t \in \mathbb{R}$.

Altogether, we will formulate all results for both solution spaces $\mathcal{S}=\mathcal{C}^{\infty}$ and $\mathcal{S}=$ $\mathbb{D}_{\mathrm{pwC}} \infty$, so that readers who feel uneasy about the distributional solution framework can ignore it.

Before stating our main results concerning the solution theory of the DAE (1.1), we need the following result about polynomial matrices. A (square) polynomial matrix 
$U(s) \in \mathbb{K}^{n \times n}[s]$ is called unimodular if and only if it is invertible within the ring $\mathbb{K}^{n \times n}[s]$, i.e., there exists $V(s) \in \mathbb{K}^{n \times n}[s]$ such that $U(s) V(s)=I$.

LEMma 3.1 (existence of unimodular inverse). Consider a matrix pencil $s E-A \in$ $\mathbb{K}^{m \times n}[s], m \neq n$, such that $\operatorname{rank} \lambda E-A=\min \{m, n\}$ for all $\lambda \in \mathbb{C}$. Then there exist polynomial matrices $M(s) \in \mathbb{K}^{n \times m}[s]$ and $K(s) \in \mathbb{K}^{n^{\prime} \times m^{\prime}}[s], n^{\prime}, m^{\prime} \in \mathbb{N}$, such that, if $m<n,[M(s), K(s)]$ is unimodular and

$$
(s E-A)[M(s), K(s)]=\left[I_{m}, 0\right],
$$

or, if $m>n,\left[\begin{array}{c}M(s) \\ K(s)\end{array}\right]$ is unimodular and

$$
\left[\begin{array}{c}
M(s) \\
K(s)
\end{array}\right](s E-A)=\left[\begin{array}{c}
I_{n} \\
0
\end{array}\right] .
$$

The proof is carried out in section 4.4.

The following theorem gives a complete characterization of the solution behavior of the DAE (1.1). Note that a discussion of the solution behavior of a DAE which is already in $\mathrm{KCF}$ is rather straightforward (see, e.g., [39]); however, a characterization based just on the QKF (2.5) without knowledge of a more detailed structure (e.g., some staircase form or even the KCF of $s E_{P}-A_{P}$ and $\left.s E_{Q}-A_{Q}\right)$ seems new.

THEOREM 3.2 (complete characterization of solutions of the DAE). Let $s E-A \in$ $\mathbb{R}^{m \times n}[s]$ and use the notation from Theorem 2.6. Consider the solution space $\mathcal{S}=\mathcal{C}^{\infty}$ or $\mathcal{S}=\mathbb{D}_{\mathrm{pwC}} \infty$ and let $f \in \mathcal{S}^{m}$. According to Theorem 2.2 , let $S_{R}, T_{R} \in \mathbf{G l}_{n_{R}}(\mathbb{R})$ be the matrices which transform $s E_{R}-A_{R}$ in quasi-Weierstraß form, i.e.,

$$
\left[\begin{array}{ccc}
I & 0 & 0 \\
0 & S_{R} & 0 \\
0 & 0 & I
\end{array}\right] S(s E-A) T\left[\begin{array}{ccc}
I & 0 & 0 \\
0 & T_{R} & 0 \\
0 & 0 & I
\end{array}\right]=\left[\begin{array}{cccc}
s E_{P}-A_{P} & 0 & 0 & 0 \\
0 & s I-J & 0 & 0 \\
0 & 0 & s N-I & 0 \\
0 & 0 & 0 & s E_{Q}-A_{Q}
\end{array}\right]
$$

and let $\left(f_{P}^{\top}, f_{J}^{\top}, f_{N}^{\top}, f_{Q}^{\top}\right)^{\top}:=\left[\begin{array}{ccc}I & 0 & 0 \\ 0 & S_{R} & 0 \\ 0 & 0 & I\end{array}\right] S f$, where the splitting corresponds to the block sizes in (3.1). According to Lemma 3.1 choose unimodular matrices $\left[M_{P}(s), K_{P}(s)\right] \in$ $\mathbb{R}^{n_{P} \times\left(m_{P}+\left(n_{P}-m_{P}\right)\right)}[s]$ and $\left[\begin{array}{l}M_{Q}(s) \\ K_{Q}(s)\end{array}\right] \in \mathbb{R}^{\left(n_{Q}+\left(m_{Q}-n_{Q}\right)\right) \times m_{Q}}[s]$ such that

$$
\left(s E_{P}-A_{P}\right)\left[M_{P}(s), K_{P}(s)\right]=[I, 0] \quad \text { and } \quad\left[\begin{array}{c}
M_{Q}(s) \\
K_{Q}(s)
\end{array}\right]\left(s E_{Q}-A_{Q}\right)=\left[\begin{array}{l}
I \\
0
\end{array}\right] .
$$

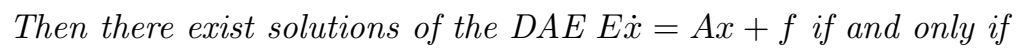

$$
K_{Q}\left(\frac{\mathrm{d}}{\mathrm{d} t}\right)\left(f_{Q}\right)=0 \text {. }
$$

If this is the case, then an initial value $x^{0}=T\left[\begin{array}{ccc}I & 0 & 0 \\ 0 & T_{R} & 0 \\ 0 & 0 & I\end{array}\right]\left(x_{P}^{0^{\top}}, x_{J}^{0^{\top}}, x_{N}^{0}{ }^{\top}, x_{Q}^{0^{\top}}\right)^{\top}$ is consistent at $t_{0} \in \mathbb{R}$, i.e., there exists a solution of the initial value problem

$$
E \dot{x}=A x+f, \quad x\left(t_{0}-\right)=x^{0},
$$

if and only if

$$
x_{Q}^{0}=\left(M_{Q}\left(\frac{\mathrm{d}}{\mathrm{d} t}\right)\left(f_{Q}\right)\right)\left(t_{0}-\right) \text { and } x_{N}^{0}=-\left(\sum_{k=0}^{n_{N}-1} N^{k}\left(\frac{\mathrm{d}}{\mathrm{d} t}\right)^{k}\left(f_{N}\right)\right)\left(t_{0}-\right) \text {. }
$$


If (3.3) holds, then any solution $x=T\left[\begin{array}{ccc}I & 0 & 0 \\ 0 & T_{R} & 0 \\ 0 & 0 & I\end{array}\right]\left(x_{P}^{\top}, x_{J}{ }^{\top}, x_{N}{ }^{\top}, x_{Q}{ }^{\top}\right)^{\top}$ of the initial value problem (3.2) has the form

$$
\begin{aligned}
x_{P} & =M_{P}\left(\frac{\mathrm{d}}{\mathrm{d} t}\right)\left(f_{P}\right)+K_{P}\left(\frac{\mathrm{d}}{\mathrm{d} t}\right)\left(u_{x_{P}^{0}}\right), \\
x_{J} & =e^{J\left(\cdot-t_{0}\right)} x_{J}^{0}+e^{J \cdot} \int_{t_{0}}\left(e^{-J \cdot} f_{J}\right), \\
x_{N} & =-\sum_{k=0}^{n_{N}-1} N^{k}\left(\frac{\mathrm{d}}{\mathrm{d} t}\right)^{k}\left(f_{N}\right), \\
x_{Q} & =M_{Q}\left(\frac{\mathrm{d}}{\mathrm{d} t}\right)\left(f_{Q}\right),
\end{aligned}
$$

where $u_{x_{P}^{0}} \in \mathcal{S}^{n_{P}-m_{P}}$ is such that the initial condition at $t_{0}$ for $x_{P}$ is satisfied (which is always possible due to Lemma 4.17), but apart from that, arbitrary.

The proof is carried out in section 5 .

Note that the antiderivative operator $\int_{t_{0}}: \mathcal{S} \rightarrow \mathcal{S}, f \mapsto F$ as used in Theorem 3.2 is uniquely defined by the two properties $\frac{\mathrm{d}}{\mathrm{d} t} F=f$ and $F\left(t_{0}-\right)=0$ (for $\mathcal{S}=\mathcal{C}^{\infty}$ this is well known and for $\mathcal{S}=\mathbb{D}_{\text {pwC }} \infty$ this is shown in [34, Prop. 3]; see also [33, Prop. 2.3.6]).

We want to use Theorem 3.2 to characterize the solutions of our example DAE (1.2). We first observe that the regular part can be brought into quasi-Weierstraß form $s 0-I$ by premultiplying with $S_{R}=A_{R}^{-1}$. In particular, the $J$-part is nonexistent, which means that the circuit contains no classical dynamics. We choose

$$
\left[M_{P}(s), K_{P}(s)\right]=\left[\begin{array}{c|cc}
0 & 1 & 0 \\
0 & 0 & 1 \\
-1 & C R s & 1
\end{array}\right] \quad \text { and } \quad\left[\begin{array}{c}
M_{Q}(s) \\
K_{Q}(s)
\end{array}\right]=\left[\begin{array}{cc}
1 & 0 \\
\hline-L K s & 1
\end{array}\right] .
$$

Furthermore, $f_{P}=\frac{V}{R_{G}+R_{F}}, f_{J}=\emptyset, f_{N}=\frac{V}{K}\left[-1,-1,-K, 0,0,-\frac{1}{R_{G}},-\frac{1}{R_{G}}, 0,-\frac{1}{R_{G}}, \frac{1}{R_{G}}\right]^{\top}$, $f_{Q}=[I, V]^{\top}, T_{R}=I$; hence the DAE (1.2) is solvable if and only if

$$
0=K_{Q}\left(\frac{\mathrm{d}}{\mathrm{d} t}\right)\left(f_{Q}\right)=-L K \frac{\mathrm{d}}{\mathrm{d} t} I+V \quad \text { or, equivalently, } V=L K \frac{\mathrm{d}}{\mathrm{d} t} I,
$$

i.e., the voltage source must be proportional to the change of current provided by the current source. In that case, the initial value must fulfill

$$
x(0-)=T\left[*, *, *,-f_{N}(0-)^{\top}, M_{Q}\left(\frac{\mathrm{d}}{\mathrm{d} t}\right)\left(f_{Q}\right)(0-)\right]^{\top},
$$

i.e., recalling that $x=\left(p_{+}, p_{-}, p_{o}, p_{T}, i_{L}, i_{p}, i_{m}, i_{G}, i_{F}, i_{R}, i_{o}, i_{V}, i_{C}, i_{T}\right)^{\top}, i_{R}(0-), i_{o}(0-)$, $i_{V}(0-)$ are arbitrary, and $p_{-}(0-)=\frac{V(0-)}{K}, p_{+}(0-)=\frac{V(0-)}{K}, p_{o}(0-)=V(0-)$, $p_{T}(0-)=R i_{R}(0-), i_{L}(0-)=I(0-), i_{p}(0-)=0, i_{m}(0-)=0, i_{G}=\frac{V(0-)}{R_{F}+R_{G}}$, $i_{F}=\frac{V(0-)}{R_{F}+R_{G}}, i_{C}(0-)=i_{V}(0-)-i_{o}(0-)+\frac{V(0-)}{R_{F}+R_{G}}, i_{T}(0-)=i_{o}(0-)-i_{R}(0-)-$ $i_{V}(0-)-\frac{V(0-)}{R_{F}+R_{G}}$. If these conditions are satisfied, then all solutions of the initial value problem corresponding to our example DAE (1.2) are given by

$$
\begin{aligned}
x & =T\left[u_{1}, u_{2}, \frac{-V}{R_{G}+R_{F}}+R C \dot{u}_{1}+u_{2}, \frac{V}{K}, \frac{V}{K}, V, 0,0, \frac{V}{R_{F}+R_{G}}, \frac{V}{R_{F}+R_{G}}, 0, \frac{V}{R_{F}+R_{G}}, \frac{-V}{R_{F}+R_{G}}, I\right]^{\top}, \\
& =\left[\frac{V}{K}, \frac{V}{K}, V, p_{T}\left(u_{1}\right), I, 0,0, \frac{V}{R_{F}+R_{G}}, \frac{V}{R_{F}+R_{G}}, i_{R}\left(u_{1}\right), i_{o}\left(u_{2}\right), i_{V}\left(u_{1}, u_{2}\right), i_{C}\left(u_{1}\right), i_{T}\left(u_{1}\right)\right]^{\top},
\end{aligned}
$$

where $u_{1}, u_{2} \in \mathcal{S}$ are arbitrary, apart from the initial conditions

$u_{1}(0-)=i_{R}(0-)-\frac{V(0-)}{R}, \dot{u}_{1}(0-)=\frac{1}{C R}\left(i_{V}(0-)+\frac{V(0-)}{R_{F}+R_{G}}-i_{o}(0-)\right), u_{2}(0-)=i_{o}(0-)$ 
and

$$
\begin{aligned}
& p_{T}\left(u_{1}\right)=V+R u_{1}, \quad i_{R}\left(u_{1}\right)=u_{1}+\frac{V}{R}, \quad i_{o}\left(u_{2}\right)=u_{2}, \\
& i_{V}\left(u_{1}, u_{2}\right)=u_{2}-\frac{V}{R_{F}+R_{G}}+C R \dot{u}_{1}, \quad i_{C}\left(u_{1}\right)=C R \dot{u}_{1}, \quad i_{T}\left(u_{1}\right)=-u_{1}-\frac{V}{R}-C R \dot{u}_{1} .
\end{aligned}
$$

Remark 3.3. A similar statement as in Theorem 3.2 is also possible if we consider only the QKTF (2.3), i.e., instead of (3.1) we consider

$$
\begin{aligned}
& {\left[\begin{array}{ccc}
I & 0 & 0 \\
0 & S_{R} & 0 \\
0 & 0 & I
\end{array}\right] S_{\text {trian }}(s E-A) T_{\text {trian }}\left[\begin{array}{ccc}
I & 0 & 0 \\
0 & T_{R} & 0 \\
0 & 0 & I
\end{array}\right]} \\
& =\left[\begin{array}{cccc}
s E_{P}-A_{P} & s E_{P J}-A_{P J} & s E_{P N}-A_{P N} & s E_{P Q}-A_{P Q} \\
0 & s I-J & 0 & s E_{J Q}-A_{J Q} \\
0 & 0 & s N-I & s E_{N Q}-A_{N Q} \\
0 & 0 & 0 & s E_{Q}-A_{Q}
\end{array}\right] .
\end{aligned}
$$

The corresponding conditions for the $Q$-part remain the same; in the condition for the $N$-part the inhomogeneity $f_{N}$ is replaced by $f_{N}-\left(E_{N Q} \frac{\mathrm{d}}{\mathrm{d} t}-A_{N Q}\right)\left(x_{Q}\right)$, in the $J$-part the inhomogeneity $f_{J}$ is replaced by $f_{J}-\left(E_{J Q} \frac{\mathrm{d}}{\mathrm{d} t}-A_{J Q}\right)\left(x_{Q}\right)$, and in the $P$-part the inhomogeneity $f_{P}$ is replaced by $f_{P}-\left(E_{P J} \frac{\mathrm{d}}{\mathrm{d} t}-A_{P J}\right)\left(x_{J}\right)-\left(E_{P N} \frac{\mathrm{d}}{\mathrm{d} t}-A_{P N}\right)\left(x_{N}\right)-$ $\left(E_{P Q} \frac{\mathrm{d}}{\mathrm{d} t}-A_{P Q}\right)\left(x_{Q}\right)$.

4. Useful lemmas. In this section we collect several lemmas which are needed to prove the main results. Since we use results from different areas we group the lemmas accordingly into subsections.

\subsection{Standard results from linear algebra.}

LEMma 4.1 (orthogonal complements and (pre-)images). For any matrix $M \in$ $\mathbb{K}^{p \times q}$ we have

(i) for all subspaces $\mathcal{S} \subseteq \mathbb{K}^{p}$ it holds that $\left(M^{-1} \mathcal{S}\right)^{\perp}=M^{\top}\left(\mathcal{S}^{\perp}\right)$;

(ii) for all subspaces $\mathcal{S} \subseteq \mathbb{K}^{q}$ it holds that $(M \mathcal{S})^{\perp}=M^{-\top}\left(\mathcal{S}^{\perp}\right)$.

Proof. Property (i) is shown, e.g., in [7, Property 3.1.3]. Property (ii) follows from considering (i) for $M^{\top}, \mathcal{S}^{\perp}$ instead of $M, \mathcal{S}$ and then taking orthogonal complements.

Lemma 4.2 (rank of matrices). Let $A, B \in \mathbb{K}^{m \times n}$ with $\operatorname{im} B \subseteq \operatorname{im} A$. Then for almost all $c \in \mathbb{K}$,

$$
\operatorname{rank} A=\operatorname{rank}(A+c B)
$$

or, equivalently,

$$
\operatorname{im} A=\operatorname{im}(A+c B) .
$$

In fact, $\operatorname{rank} A<\operatorname{rank}(A+c B)$ can only hold for at most $r=\operatorname{rank} A$ many values of c.

Proof. Consider the Smith form [32] of $A$,

$$
U A V=\left[\begin{array}{cc}
\Sigma_{r} & 0 \\
0 & 0
\end{array}\right]
$$

with invertible matrices $U \in \mathbb{K}^{m \times m}$ and $V \in \mathbb{K}^{n \times n}$ and $\Sigma_{r}=\operatorname{diag}\left(\sigma_{1}, \sigma_{2}, \ldots, \sigma_{r}\right)$, $\sigma_{i} \in \mathbb{K} \backslash\{0\}, r=\operatorname{rank} A$. Write

$$
U B V=\left[\begin{array}{ll}
B_{11} & B_{12} \\
B_{21} & B_{22}
\end{array}\right]
$$


where $B_{11} \in \mathbb{K}^{r \times r}$. Since $\operatorname{im} B \subseteq \operatorname{im} A$, it follows that $B_{21}=0$ and $B_{22}=0$. Hence, we obtain the following implications:

$$
\begin{aligned}
\operatorname{rank}(A+c B)<\operatorname{rank} A \Rightarrow \operatorname{rank}\left[\Sigma_{r}+c B_{11}, c B_{12}\right]<\operatorname{rank}\left[\Sigma_{r}, 0\right]=r \\
\quad \Rightarrow \operatorname{rank}\left(\Sigma_{r}+c B_{11}\right)<r \Rightarrow \operatorname{det}\left(\Sigma_{r}+c B_{11}\right)=0 .
\end{aligned}
$$

Since $\operatorname{det}\left(\Sigma_{r}+c B_{11}\right)$ is a polynomial in $c$ of degree at most $r$ but not the zero polynomial (since $\operatorname{det}\left(\Sigma_{r}\right) \neq 0$ ), it can have at most $r$ zeros. This proves the claim.

Lemma 4.3 (dimension formulae). Let $\mathcal{S} \subseteq \mathbb{K}^{n}$ be any linear subspace of $\mathbb{K}^{n}$ and $M \in \mathbb{K}^{m \times n}$. Then

$$
\operatorname{dim} M \mathcal{S}=\operatorname{dim} \mathcal{S}-\operatorname{dim}(\operatorname{ker} M \cap \mathcal{S})
$$

Furthermore, for any two linear subspaces $\mathcal{S}, \mathcal{T}$ of $\mathbb{K}^{n}$ we have

$$
\operatorname{dim}(\mathcal{S}+\mathcal{T})=\operatorname{dim} \mathcal{S}+\operatorname{dim} \mathcal{T}-\operatorname{dim}(\mathcal{S} \cap \mathcal{T})
$$

Proof. See any textbook on linear algebra for the proof.

4.2. The Wong sequences. The next lemma highlights an important property of the intersection of the limits of the Wong sequences.

Lemma 4.4 (property of $\mathcal{V}^{*} \cap \mathcal{W}^{*}$ ). Let $s E-A \in \mathbb{K}^{m \times n}[s]$ and $\mathcal{V}^{*}, \mathcal{W}^{*}$ be the limits of the corresponding Wong sequences. Then

$$
E\left(\mathcal{V}^{*} \cap \mathcal{W}^{*}\right)=E \mathcal{V}^{*} \cap A \mathcal{W}^{*}=A\left(\mathcal{V}^{*} \cap \mathcal{W}^{*}\right)
$$

Proof. Clearly, invoking (2.2),

$E\left(\mathcal{V}^{*} \cap \mathcal{W}^{*}\right) \subseteq E \mathcal{V}^{*} \cap E \mathcal{W}^{*} \subseteq E \mathcal{V}^{*} \cap A \mathcal{W}^{*}$ and $A\left(\mathcal{V}^{*} \cap \mathcal{W}^{*}\right) \subseteq A \mathcal{V}^{*} \cap A \mathcal{W}^{*} \subseteq E \mathcal{V}^{*} \cap A \mathcal{W}^{*}$

hence it remains to show the converse subspace relationship. To this end we choose $x \in E \mathcal{V}^{*} \cap A \mathcal{W}^{*}$, which implies the existence of $v \in \mathcal{V}^{*}$ and $w \in \mathcal{W}^{*}$ such that

$$
E v=x=A w
$$

hence

$$
v \in E^{-1}\{A w\} \subseteq E^{-1}\left(A \mathcal{W}^{*}\right)=\mathcal{W}^{*}, \quad w \in A^{-1}\{E v\} \subseteq A^{-1}\left(E \mathcal{V}^{*}\right)=\mathcal{V}^{*} .
$$

Therefore $v, w \in \mathcal{V}^{*} \cap \mathcal{W}^{*}$ and $x=E v \in E\left(\mathcal{V}^{*} \cap \mathcal{W}^{*}\right)$ as well as $x=A w \in A\left(\mathcal{V}^{*} \cap \mathcal{W}^{*}\right)$, which concludes the proof.

For the proof of the main result we briefly consider the Wong sequences of the (conjugate) transposed matrix pencil $s E^{\top}-A^{\top}$; these are connected to the original Wong sequences as follows.

Lemma 4.5 (Wong sequences of the transposed matrix pencil). Consider a matrix pencil $s E-A \in \mathbb{K}^{m \times n}[s]$ with corresponding limits of the Wong sequences $\mathcal{V}^{*}$ and $\mathcal{W}^{*}$. Denote with $\widehat{\mathcal{V}}^{*}$ and $\widehat{\mathcal{W}}^{*}$ the limits of the Wong sequences of the (conjugate) transposed matrix pencil $s E^{\top}-A^{\top}$. Then the following holds:

$$
\widehat{\mathcal{W}}^{*}=\left(E \mathcal{V}^{*}\right)^{\perp} \quad \text { and } \quad \widehat{\mathcal{V}}^{*}=\left(A \mathcal{W}^{*}\right)^{\perp} .
$$

Proof. We show that for all $i \in \mathbb{N}$,

$$
\left(E \mathcal{V}_{i}\right)^{\perp}=\widehat{\mathcal{W}}_{i+1} \quad \text { and } \quad\left(A \mathcal{W}_{i}\right)^{\perp}=\widehat{\mathcal{V}}_{i}
$$


from which the claim follows. For $i=0$ this follows from

$$
\left(E \mathcal{V}_{0}\right)^{\perp}=(\operatorname{im} E)^{\perp}=\operatorname{ker} E^{\top}=E^{-\top}\left(A^{\top}\{0\}\right)=\widehat{\mathcal{W}}_{1}
$$

and

$$
\left(A \mathcal{W}_{0}\right)^{\perp}=\{0\}^{\perp}=\mathbb{R}^{m}=\widehat{\mathcal{V}}_{0} .
$$

Now suppose that (4.1) holds for some $i \in \mathbb{N}$. Then

$$
\begin{aligned}
& \left(E \mathcal{V}_{i+1}\right)^{\perp} \quad=\quad\left(E A^{-1}\left(E \mathcal{V}_{i}\right)\right)^{\perp} \\
& \stackrel{\text { Lem. }}{=}{ }^{.1(\mathrm{ii})} E^{-\top}\left(A^{-1}\left(E \mathcal{V}_{i}\right)\right)^{\perp} \\
& \stackrel{\text { Lem. }}{=}{ }^{4.1(\mathrm{i})} E^{-\top}\left(A^{\top}\left(E \mathcal{V}_{i}\right)^{\perp}\right) \\
& =E^{-\top}\left(A^{\top} \widehat{\mathcal{W}}_{i+1}\right)=\widehat{\mathcal{W}}_{i+2},
\end{aligned}
$$

and analogously it follows that $\left(A \mathcal{W}_{i+1}\right)^{\perp}=\widehat{\mathcal{V}}_{i+1}$; hence we have inductively shown (4.1).

4.3. Singular chains. In this subsection we introduce the notion of singular chains for matrix pencils. This notion is inspired by the theory of linear relations (see [30]), where they are a vital tool for analyzing the structure of linear relations. They also play an important role in former works on the KCF; see, e.g., [16, Chap. XII] and [3]. However, in these works only singular chains of minimal length are considered. We use them here to determine the structure of the intersection $\mathcal{V}^{*} \cap \mathcal{W}^{*}$ of the limits of the Wong sequences.

Definition 4.6 (singular chain). Let $s E-A \in \mathbb{K}^{m \times n}[s]$. For $k \in \mathbb{N}$ the tuple $\left(x_{0}, \ldots, x_{k}\right) \in\left(\mathbb{K}^{n}\right)^{k+1}$ is called a singular chain of the matrix pencil $s E-A$ if and only if

$$
0=A x_{0}, E x_{0}=A x_{1}, \ldots, E x_{k-1}=A x_{k}, E x_{k}=0
$$

or, equivalently, the polynomial vector $x(s)=x_{0}+x_{1} s+\cdots+x_{k} s^{k} \in \mathbb{K}^{n}[s]$ satisfies $(s E-A) x(s)=0$.

Note that with every singular chain $\left(x_{0}, x_{1}, \ldots, x_{k}\right)$ also the tuple $\left(0, \ldots, 0, x_{0}, \ldots\right.$, $\left.x_{k}, 0, \ldots, 0\right)$ is a singular chain of $s E-A$. Furthermore, with every singular chain, each scalar multiple is a singular chain and for two singular chains of the same length the sum of both is a singular chain. A singular chain $\left(x_{0}, \ldots, x_{k}\right)$ is called linearly independent if the vectors $x_{0}, \ldots, x_{k}$ are linearly independent.

Lemma 4.7 (linear independency of singular chains). Let $s E-A \in \mathbb{K}^{m \times n}[s]$. For every nontrivial singular chain $\left(x_{0}, x_{1}, \ldots, x_{k}\right), k \in \mathbb{N}$, of $s E-A$ there exists $\ell \in \mathbb{N}, \ell \leq k$, and a linearly independent singular chain $\left(y_{0}, y_{1}, \ldots, y_{\ell}\right)$ with $\operatorname{span}\left\{x_{0}, x_{1}, \ldots, x_{k}\right\}=\operatorname{span}\left\{y_{0}, y_{1}, \ldots, y_{\ell}\right\}$.

Proof. This result is an extension of [30, Lem. 3.1]; hence our proof resembles some ideas of the latter.

If $\left(x_{0}, x_{1}, \ldots, x_{k}\right)$ is already a linearly independent singular chain, then nothing is to show, therefore, assume existence of a minimal $\ell \in\{0,1, \ldots, k-1\}$ such that 
$x_{\ell+1}=\sum_{i=0}^{\ell} \alpha_{i} x_{i}$ for some $\alpha_{i} \in \mathbb{K}, i=0, \ldots, \ell$. Consider the chains

$$
\begin{aligned}
& \alpha_{0}\left(0, \quad 0, \ldots, \quad 0, \quad 0, \quad x_{0}, \quad x_{1}, \ldots, \quad x_{\ell}, x_{\ell+1}, \ldots, x_{k-1}, x_{k}\right), \\
& \alpha_{1}\left(0,0, \ldots, \quad 0, \quad x_{0}, \quad x_{1}, \ldots, \quad x_{\ell}, x_{\ell+1}, \ldots, x_{k-1}, x_{k}, 0\right) \text {, } \\
& \alpha_{2}\left(0,0, \ldots, x_{0}, x_{1}, \ldots, x_{\ell}, x_{\ell+1}, \ldots, x_{k-1}, x_{k}, 0,0\right) \text {, } \\
& \alpha_{\ell-1}\left(0, x_{0}, x_{1}, \ldots, x_{\ell-2}, x_{\ell-1}, x_{\ell}, x_{\ell+1}, \ldots x_{k}, \quad 0, \ldots, 0\right),
\end{aligned}
$$

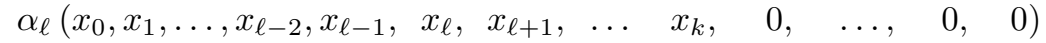

and denote its sum by $\left(z_{0}, z_{1}, \ldots, z_{k+\ell}\right)$. Note that by construction $z_{\ell}=\sum_{i=0}^{\ell} \alpha_{i} x_{i}=$ $x_{\ell+1}$. Now consider the singular chain $\left(v_{0}, v_{1}, \ldots, v_{k+\ell+1}\right):=\left(x_{0}, x_{1}, \ldots, x_{k}, 0, \ldots, 0\right)-$ $\left(0, z_{0}, z_{1}, \ldots, z_{\ell+k}\right)$, which has the property that $v_{\ell+1}=x_{\ell+1}-z_{\ell}=0$. In particular $\left(v_{0}, v_{1}, \ldots, v_{\ell}\right)$ and $\left(v_{\ell+2}, v_{\ell+3}, \ldots, v_{k+\ell+1}\right)$ are both singular chains. Furthermore (we abbreviate $\alpha_{i} I$ with $\alpha_{i}$ ),

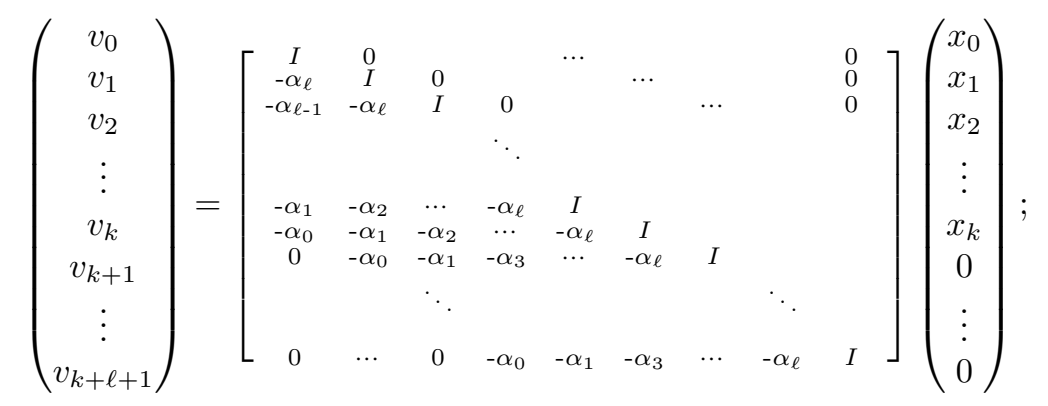

hence $\operatorname{span}\left\{v_{0}, v_{1}, \ldots, v_{k+\ell+1}\right\}=\operatorname{span}\left\{x_{0}, x_{1}, \ldots, x_{k}\right\}=\operatorname{span}\left\{v_{0}, v_{1}, \ldots, v_{k}\right\}$. In particular

$$
\operatorname{span}\left\{v_{k+1}, v_{k+2}, \ldots, v_{k+\ell+1}\right\} \subseteq \operatorname{span}\left\{v_{0}, v_{1}, \ldots, v_{k}\right\} ;
$$

hence, by applying Lemma 4.2 , there exists $c \neq 0$ such that (note that $\ell<k$ )

$$
\operatorname{im}\left[v_{0}, v_{1}, \ldots, v_{k}\right]=\operatorname{im}\left(\left[v_{0}, v_{1}, \ldots, v_{k}\right]+c\left[v_{k+1}, v_{k+2}, \ldots, v_{k+\ell+1}, 0, \ldots, 0\right]\right) .
$$

Therefore, the singular chain

$\left(w_{0}, w_{1}, \ldots, w_{k-1}\right):=c\left(v_{\ell+2}, \ldots, v_{k}, v_{k+1}, v_{k+2}, \ldots, v_{k+\ell+1}\right)+\left(0, \ldots, 0, v_{0}, v_{1}, \ldots, v_{\ell}\right)$

has the property

$$
\begin{aligned}
\operatorname{span}\left\{w_{0}, w_{1}, \ldots, w_{k-1}\right\}= & \operatorname{span}\left\{v_{\ell+2}, v_{\ell+3}, \ldots, v_{k}\right\} \\
& +\operatorname{span}\left\{c v_{k+1}+v_{0}, c v_{k+2}+v_{1}, \ldots, c v_{k+\ell+1}+v_{\ell}\right\} \\
\stackrel{v_{\ell+1}=0}{=} & \operatorname{im}\left(\left[v_{0}, v_{1}, \ldots, v_{k}\right]+c\left[v_{k+1}, v_{k+2}, \ldots, v_{k+\ell+1}, 0, \ldots, 0\right]\right) \\
\stackrel{(4.2)}{=} & \operatorname{im}\left[v_{0}, v_{1}, \ldots, v_{k}\right] \\
= & \operatorname{span}\left\{v_{0}, v_{1}, \ldots, v_{k}\right\}=\operatorname{span}\left\{x_{0}, x_{1}, \ldots, x_{k}\right\} .
\end{aligned}
$$

Altogether, we have obtained a shorter singular chain which spans the same subspace as the original singular chain. Repeating this procedure until one obtains a linearly independent singular chain proves the claim. 
COROLlary 4.8 (basis of the singular chain manifold). Consider a matrix pencil $s E-A \in \mathbb{K}^{m \times n}[s]$ and let the singular chain manifold be given by

$\mathcal{K}:=\left\{x \in \mathbb{R}^{n} \mid \exists k, i \in \mathbb{N} \exists\right.$ sing. chain $\left.\left(x_{0}, \ldots, x_{i-1}, x=x_{i}, x_{i+1}, \ldots, x_{k}\right) \in\left(\mathbb{K}^{n}\right)^{k+1}\right\}$,

i.e., $\mathcal{K}$ is the set of all vectors $x$ appearing somewhere in some singular chain of $s E-A$. Then there exists a linearly independent singular chain $\left(x_{0}, x_{1}, \ldots, x_{k}\right)$ of $s E-A$ such that

$$
\mathcal{K}=\operatorname{span}\left\{x_{0}, \ldots, x_{k}\right\} .
$$

Proof. First note that $\mathcal{K}$ is indeed a linear subspace of $\mathbb{K}^{n}$, since the scalar multiple of every chain is also a chain and the sum of two chains (extending the chains appropriately with zero vectors) is again a chain.

Let $y^{0}, y^{1}, \ldots, y^{\ell}$ be any basis of $\mathcal{K}$. By the definition of $\mathcal{K}$, for each $i=0,1, \ldots, \ell$ there exist chains $\left(y_{0}^{i}, y_{1}^{i}, \ldots, y_{k_{i}}^{i}\right)$, which contain $y^{i}$. Let $\left(v_{0}, v_{1}, \ldots, v_{\hat{k}}\right)$ with $\hat{k}=k_{0}+$ $k_{1}+\ldots+k_{\ell}$ being the chain which results by concatenating the chains $\left(y_{0}^{i}, y_{1}^{i}, \ldots, y_{k_{i}}^{i}\right)$. Clearly, $\operatorname{span}\left\{v_{0}, \ldots, v_{\hat{k}}\right\}=\mathcal{K}$; hence, Lemma 4.7 yields the claim.

The following result can, in substance, be found in [3]. However, the proof therein is difficult to follow, involving quotient spaces and additional sequences of subspaces. Our presentation is much more straightforward and simpler.

Lemma 4.9 (singular chain manifold and the Wong sequences). Consider a matrix pencil $s E-A \in \mathbb{K}^{m \times n}[s]$ with the limits $\mathcal{V}^{*}$ and $\mathcal{W}^{*}$ of the Wong sequences. Let the singular chain manifold $\mathcal{K}$ be given as in Corollary 4.8; then

$$
\mathcal{V}^{*} \cap \mathcal{W}^{*}=\mathcal{K} .
$$

Proof. Step 1. We show $\mathcal{K} \subseteq \mathcal{V}^{*} \cap \mathcal{W}^{*}$.

Let $\left(x_{0}, \ldots, x_{k}\right)$ be a singular chain. Clearly we have $x_{0} \in A^{-1}(E\{0\})=\operatorname{ker} A \subseteq$ $\mathcal{V}^{*}$ and $x_{k} \in E^{-1}(A\{0\})=\operatorname{ker} E \subseteq \mathcal{W}^{*}$; hence, inductively we have, for $i=$ $0,1, \ldots, k-1$ and $j=k, k-1, \ldots, 1$,

$x_{i+1} \in A^{-1}\left(E\left\{x_{i}\right\}\right) \subseteq A^{-1}\left(E \mathcal{V}^{*}\right)=\mathcal{V}^{*}$ and $x_{j-1} \in E^{-1}\left(A\left\{x_{j}\right\}\right) \subseteq E^{-1}\left(A \mathcal{W}^{*}\right)=\mathcal{W}^{*}$.

Therefore,

$$
x_{0}, \ldots, x_{k} \in \mathcal{V}^{*} \cap \mathcal{W}^{*}
$$

Step 2. We show $\mathcal{V}^{*} \cap \mathcal{W}^{*} \subseteq \mathcal{K}$.

Let $x \in \mathcal{V}^{*} \cap \mathcal{W}^{*}$, in particular, $x \in \mathcal{W}^{*}=\mathcal{W}_{l^{*}}$ for some $l^{*} \in \mathbb{N}$. Hence there exists $x_{1} \in \mathcal{W}_{l^{*}-1}, x_{2} \in \mathcal{W}_{l^{*}-2}, \ldots, x_{l^{*}} \in \mathcal{W}_{0}=\{0\}$, such that, for $x_{0}:=x$,

$$
E x_{0}=A x_{1}, \quad E x_{1}=A x_{2}, \ldots, E x_{l^{*}-1}=A x_{l^{*}}, \quad E x_{l^{*}}=0 .
$$

Furthermore, since, by Lemma $4.4, E\left(\mathcal{V}^{*} \cap \mathcal{W}^{*}\right)=A\left(\mathcal{V}^{*} \cap \mathcal{W}^{*}\right)$, there exist $x_{-1}, x_{-2}, \ldots$, $x_{-\left(l^{*}+1\right)} \in \mathcal{V}^{*} \cap \mathcal{W}^{*}$ such that

$$
A x_{0}=E x_{-1}, A x_{-1}=E x_{-2}, \ldots, A x_{-\left(l^{*}-1\right)}=E x_{-l^{*}}, A x_{-l^{*}}=E x_{-\left(l^{*}+1\right)} .
$$

Let $\tilde{x}_{-\left(l^{*}+1\right)}:=-x_{-\left(l^{*}+1\right)} \in \mathcal{V}^{*} \cap \mathcal{W}^{*} \subseteq \mathcal{W}^{*}$; then (with the same argument as above) there exist $\tilde{x}_{-l^{*}}, \tilde{x}_{-\left(l^{*}-1\right)}, \ldots, \tilde{x}_{-1} \in \mathcal{W}^{*}$ such that

$$
E \tilde{x}_{-\left(l^{*}+1\right)}=A \tilde{x}_{-l^{*}}, \quad E \tilde{x}_{-l^{*}}=A \tilde{x}_{-\left(l^{*}-1\right)}, \ldots, E \tilde{x}_{-2}=A \tilde{x}_{-1}, \quad E \tilde{x}_{-1}=0,
$$


and thus, defining $\hat{x}_{-i}=x_{-i}+\tilde{x}_{-i}$, for $i=1, \ldots, l^{*}+1$, we have $\hat{x}_{-\left(l^{*}+1\right)}=0$ and we get

$$
0=E \hat{x}_{-\left(l^{*}+1\right)}=A \hat{x}_{-l^{*}}, E \hat{x}_{-l^{*}}=A \hat{x}_{-\left(l^{*}-1\right)}, \ldots, E \hat{x}_{-2}=A \hat{x}_{-1}, E \hat{x}_{-1}=E x_{-1}=A x_{0} .
$$

This shows that $\left(\hat{x}_{-l^{*}}, \hat{x}_{-\left(l^{*}-1\right)}, \ldots, \hat{x}_{-1}, x_{0}, x_{1}, \ldots, x_{l^{*}}\right)$ is a singular chain and $x=$ $x_{0} \in \mathcal{K}$.

The last result in this section relates singular chains with the column rank of the matrix pencil $s E-A$.

Lemma 4.10 (column rank deficit implies singular chains). Let $s E-A \in$ $\mathbb{K}^{m \times n}[s]$. If $\operatorname{rank}_{\mathbb{C}}(\lambda E-A)<n$ for all $\lambda \in \mathbb{C} \cup\{\infty\}$, then there exists a nontrivial singular chain of $s E-A$.

Proof. It suffices to observe that Definition 4.6 coincides (modulo a reversed indexing) with the notion of singular chains in [30] applied to the linear relation $E^{-1} A:=\left\{(x, y) \in \mathbb{K}^{n} \times \mathbb{K}^{n} \mid A x=E y\right\}$. Then the claim follows for $\mathbb{K}=\mathbb{C}$ from [30, Thm. 4.4]. The main idea of the proof there is to choose any $m+1$ different eigenvalues and corresponding eigenvectors. This is also possible for $\mathbb{K}=\mathbb{R}$ and $\mathbb{K}=\mathbb{Q}$; hence the proof in [30] is also valid for $\mathbb{K}=\mathbb{R}$ and $\mathbb{K}=\mathbb{Q}$.

4.4. Polynomial matrices. In the following we will say that $P(s) \in \mathbb{K}^{m \times n}[s]$ can be extended to a unimodular matrix if and only if, in the case $m<n$, there exists $Q(s) \in \mathbb{K}^{n-m \times n}[s]$ such that $\left[\begin{array}{l}P(s) \\ Q(s)\end{array}\right]$ is unimodular, in the case $m>n$, there exists $Q(s) \in \mathbb{K}^{m \times m-n}[s]$ such that $[P(s), Q(s)]$ is unimodular, and, in the case $m=n$, $P(s)$ itself is unimodular.

Lemma 4.11 (unimodular extension). A matrix $P(s) \in \mathbb{K}^{m \times n}[s]$ can be extended to a unimodular matrix if and only if $\operatorname{rank}_{\mathbb{C}} P(\lambda)=\min \{m, n\}$ for all $\lambda \in \mathbb{C}$.

Proof. Necessity is clear; hence it remains to show that under the full rank assumption a unimodular extension is possible. Note that $\mathbb{K}[s]$ is a principal ideal domain; hence we can consider the Smith normal form [32] of $P(s)$ given by

$$
P(s)=U(s)\left[\begin{array}{cc}
\Sigma_{r}(s) & 0 \\
0 & 0
\end{array}\right] V(s)
$$

where $U(s), V(s)$ are unimodular matrices and $\Sigma(s)=\operatorname{diag}\left(\sigma_{1}(s), \ldots, \sigma_{r}(s)\right), r \in \mathbb{N}$, with nonzero diagonal entries. Note that $\operatorname{rank}_{\mathbb{C}} P(\lambda)=\operatorname{rank}_{\mathbb{C}} \Sigma(\lambda)$ for all $\lambda \in \mathbb{C}$; hence the full rank condition implies $r=\min \{m, n\}$ and $\sigma_{1}(s), \ldots, \sigma_{r}(s)$ are constant (nonzero) polynomials. For $m=n$ this already shows the claim. For $m>n$, i.e., $P(s)=U(s)\left[\begin{array}{c}\Sigma_{n}(s) \\ 0\end{array}\right] V(s)$, the sought unimodular extension is given by

$$
[P(s), Q(s)]=U(s)\left[\begin{array}{c|c}
\Sigma_{n}(s) & 0 \\
0 & I
\end{array}\right]\left[\begin{array}{cc}
V(s) & 0 \\
0 & I
\end{array}\right]
$$

and, for $m<n$,

$$
\left[\begin{array}{l}
P(s) \\
Q(s)
\end{array}\right]=\left[\begin{array}{cc}
U(s) & 0 \\
0 & I
\end{array}\right]\left[\begin{array}{cc}
\Sigma_{m}(s) & 0 \\
0 & I
\end{array}\right] V(s)
$$

Proof of Lemma 3.1. Let $Q(s)$ be any unimodular extension of $s E-A$ according to Lemma 4.11. If $m<n$, choose $[M(s), K(s)]=\left[\begin{array}{c}s E-A \\ Q(s)\end{array}\right]^{-1}$, and if $m>n$, let $\left[\begin{array}{l}M(s) \\ K(s)\end{array}\right]:=[s E-A, Q(s)]^{-1}$. 
4.5. KCF for full rank pencils. In order to derive the KCF as a corollary of the QKF and also for the proof of the solvability of (2.4), we need the following lemma, which shows how to obtain the KCF for the special case of full rank pencils.

LEMma 4.12 (KCF of full rank rectangular pencil, $m<n$ case). Let $s E-A \in$ $\mathbb{K}^{m \times n}[s]$ be such that $m<n$, and let $l:=n-m$. Then $\operatorname{rank}_{\mathbb{C}}(\lambda E-A)=m$ for all $\lambda \in \mathbb{C} \cup\{\infty\}$ if and only if there exist numbers $\varepsilon_{1}, \ldots, \varepsilon_{l} \in \mathbb{N}$ and matrices $S \in \mathbf{G l}_{m}(\mathbb{K}), T \in \mathbf{G l}_{n}(\mathbb{K})$ such that

$$
S(s E-A) T=\operatorname{diag}\left(\mathcal{P}_{\varepsilon_{1}}(s), \ldots, \mathcal{P}_{\varepsilon_{l}}(s)\right),
$$

where $\mathcal{P}_{\varepsilon}(s), \varepsilon \in \mathbb{N}$, is as in Corollary 2.8.

Proof. Sufficiency is clear; hence it remains to show necessity.

If $m=0$ and $n>0$, then nothing is to show since $s E-A$ is already in the "diagonal form" with $\varepsilon_{1}=\varepsilon_{2}=\cdots=\varepsilon_{l}=0$. Hence assume $m>0$ in the following. The main idea is to reduce the problem to a smaller pencil $s E^{\prime}-A^{\prime} \in \mathbb{K}^{m^{\prime} \times n^{\prime}}[s]$ with $\operatorname{rank}_{\mathbb{C}}\left(\lambda E^{\prime}-A^{\prime}\right)=m^{\prime}<n^{\prime}<n$ for all $\lambda \in \mathbb{C} \cup\{\infty\}$. Then we can inductively use the transformation to the desired block diagonal structure for the smaller pencil to obtain the block diagonal structure for the original pencil.

By assumption $E$ does not have full column rank; hence there exists a column operation $T_{1} \in \mathbf{G l}_{n}(\mathbb{K})$ such that

$$
E T_{1}=\left[\begin{array}{cccc}
0 & * & \cdots & * \\
\vdots & \vdots & & \vdots \\
0 & * & \cdots & *
\end{array}\right] .
$$

There are two cases now: Either the first column of $A T_{1}$ is zero or it is not. We consider the two cases separately.

Case 1. The first column of $A T_{1}$ is zero.

Let $E T_{1}=:\left[0, E^{\prime}\right]$ and $A T_{1}=:\left[0, A^{\prime}\right]$. Then, clearly, $\operatorname{rank}_{\mathbb{C}}(\lambda E-A)=\operatorname{rank}_{\mathbb{C}}\left(\lambda E^{\prime}-\right.$ $\left.A^{\prime}\right)=m^{\prime}:=m$ for all $\lambda \in \mathbb{C} \cup\{\infty\}$. Furthermore, with $n^{\prime}:=n-1$, it follows that $n^{\prime} \geq m^{\prime}$. Seeking a contradiction, assume $n^{\prime}=m^{\prime}$. Then the full rank matrix $E^{\prime}$ is square and hence invertible. Let $\lambda \in \mathbb{C}$ be any eigenvalue of the matrix $E^{\prime-1} A^{\prime}$. Thus $0=\operatorname{det}\left(\lambda I-E^{\prime-1} A^{\prime}\right)=\operatorname{det}\left(E^{\prime}\right)^{-1} \operatorname{det}\left(\lambda E^{\prime}-A^{\prime}\right)$, and hence $\operatorname{rank}_{\mathbb{C}}\left(\lambda E^{\prime}-A^{\prime}\right)<m^{\prime}$, a contradiction. Altogether, this shows that $s E^{\prime}-A^{\prime} \in \mathbb{K}^{m^{\prime} \times n^{\prime}}[s]$ is a smaller pencil which satisfies the assumption of the lemma; hence we can inductively use the result of the lemma for $s E^{\prime}-A^{\prime}$ with transformation matrices $S^{\prime}$ and $T^{\prime}$. Let $S:=S^{\prime}$ and $T:=T_{1}\left[\begin{array}{cc}1 & 0 \\ 0 & T^{\prime}\end{array}\right]$; then $S(s E-A) T$ has the desired block diagonal structure which coincides with the block structure of $s E^{\prime}-A^{\prime}$ apart from one additional $\mathcal{P}_{0}$ block.

Case 2. The first column of $A T_{1}$ is not zero.

Then there exists a row operation $S_{1} \in \mathbf{G} \mathbf{l}_{m}(\mathbb{K})$ such that

$$
S_{1}\left(A T_{1}\right)=\left[\begin{array}{ccccc}
1 & * & * & \cdots & * \\
0 & * & * & \cdots & * \\
\vdots & \vdots & & & \vdots \\
0 & * & * & \cdots & *
\end{array}\right]
$$

Since $E$ has full row rank, the first row of $S_{1} E T_{1}$ cannot be the zero row; hence, there exists a second column operation $T_{2} \in \mathbf{G l}_{n}(n)$ which does not change the first 
column such that

$$
\left(S_{1} E T_{1}\right) T_{2}=\left[\begin{array}{ccccc}
0 & 1 & 0 & \cdots & 0 \\
0 & * & * & \cdots & * \\
\vdots & \vdots & & & \vdots \\
0 & * & * & \cdots & *
\end{array}\right]
$$

Now let $T_{3} \in \mathbf{G l}_{n}(\mathbb{K})$ be a column operation which adds multiples of the first column to the remaining columns such that

$$
\left(S_{1} A T_{1} T_{2}\right) T_{3}=\left[\begin{array}{ccccc}
1 & 0 & 0 & \cdots & 0 \\
0 & * & * & \cdots & * \\
\vdots & \vdots & & & \vdots \\
0 & * & * & \cdots & *
\end{array}\right]
$$

Since the first column of $S_{1} E T_{1} T_{2}$ is zero, the column operation $T_{3}$ has no effect on the matrix $S_{1} E T_{1} T_{2}$. Let

$$
S_{1} E T_{1} T_{2} T_{3}=:\left[\begin{array}{c|cccc}
0 & 1 & 0 & \cdots & 0 \\
\hline 0 & & & & \\
\vdots & & & E^{\prime} & \\
0 & & & &
\end{array}\right] \text { and } S_{1} A T_{1} T_{2} T_{3}=:\left[\begin{array}{c|cccc}
1 & 0 & 0 & \cdots & 0 \\
\hline 0 & & & & \\
\vdots & & & A^{\prime} & \\
0 & & &
\end{array}\right] \text {, }
$$

with $s E^{\prime}-A^{\prime} \in \mathbb{K}^{m^{\prime} \times n^{\prime}}[s]$ and $m^{\prime}:=m-1, n^{\prime}:=n-1$, in particular $m^{\prime}<n^{\prime}$. Seeking a contradiction, assume $\operatorname{rank}_{\mathbb{C}} \lambda E^{\prime}-A^{\prime}<m^{\prime}$ for some $\lambda \in \mathbb{C} \cup\{\infty\}$. If $\lambda=\infty$, then this implies that $E^{\prime}$ does not have full row rank, which would also imply that $E$ does not have full row rank, which is not the case. Hence we may choose a vector $v^{\prime} \in \mathbb{C}^{m^{\prime}}$ such that $v^{\prime}\left(\lambda E^{\prime}-A^{\prime}\right)=0$. Let $v:=\left[0, v^{\prime}\right] S_{1}$. Then a simple calculation reveals $v(\lambda E-A)=\left[0, v^{\prime}\left(\lambda E^{\prime}-A^{\prime}\right)\right]\left(T_{1} T_{2} T_{3}\right)^{-1}=0$, which contradicts full complex rank of $\lambda E-A$. As in the first case we can now inductively use the result of the lemma for the smaller matrix pencil $s E^{\prime}-A^{\prime}$ to obtain transformations $S^{\prime}$ and $T^{\prime}$ which put $s E^{\prime}-A^{\prime}$ in the desired block diagonal form. With $S:=\left[\begin{array}{cc}1 & 0 \\ 0 & S^{\prime}\end{array}\right] S_{1}$ and $T:=T_{1} T_{2} T_{3}\left[\begin{array}{cc}1 & 0 \\ 0 & T^{\prime}\end{array}\right]$ we obtain the same block diagonal structure for $s E-A$ as for $s E^{\prime}-A^{\prime}$ apart from the first block, which is $\mathcal{P}_{\varepsilon_{1}+1}$ instead of $\mathcal{P}_{\varepsilon_{1}}$.

The following corollary follows directly from Lemma 4.12 by transposing the respective matrices.

COROLlary 4.13 (KCF of full rank rectangular pencils, $m>n$ case). Let $s E-A \in \mathbb{K}^{m \times n}[s]$ be such that $m>n$, and let $l:=m-n$. Then $\operatorname{rank}_{\mathbb{C}}(\lambda E-A)=n$ for all $\lambda \in \mathbb{C} \cup\{\infty\}$ if and only if there exist numbers $\eta_{1}, \ldots, \eta_{l} \in \mathbb{N}$ and matrices $S \in \mathbf{G l}_{m}(\mathbb{K}), T \in \mathbf{G l}_{n}(\mathbb{K})$ such that

$$
S(s E-A) T=\operatorname{diag}\left(\mathcal{Q}_{\eta_{1}}(s), \ldots, \mathcal{Q}_{\eta_{l}}(s)\right),
$$

where $\mathcal{Q}_{\eta}(s), \eta \in \mathbb{N}$, is as in Corollary 2.8 .

4.6. Solvability of linear matrix equations. In generalization of the method presented in [11, sect. 6] we reduce the problem of solvability of (2.4) to the problem of solving a generalized Sylvester equation

$$
A X B-C X D=E .
$$


To this end the following lemma is crucial.

Lemma 4.14. Let $A, C \in \mathbb{K}^{m \times n}, B, D \in \mathbb{K}^{p \times q}, E, F \in \mathbb{K}^{m \times q}$, and consider the system of matrix equations with "unknowns" $Y \in \mathbb{K}^{n \times q}$ and $Z \in \mathbb{K}^{m \times p}$,

$$
\begin{aligned}
& 0=E+A Y+Z D, \\
& 0=F+C Y+Z B .
\end{aligned}
$$

Suppose there exists $\lambda \in \mathbb{K}$ and $M_{\lambda} \in \mathbb{K}^{q \times p}$ such that $M_{\lambda}(B-\lambda D)=I$, in particular $p \geq q$. Then, for any solution $X \in \mathbb{K}^{n \times p}$ of the matrix equation

$$
A X B-C X D=-E-(\lambda E-F) M_{\lambda} D,
$$

the matrices

$$
\begin{aligned}
& Y=X(B-\lambda D), \\
& Z=-(C-\lambda A) X-(F-\lambda E) M_{\lambda}
\end{aligned}
$$

solve (4.4).

Proof. We calculate

$$
\begin{aligned}
E+A Y+Z D & =E+A X(B-\lambda D)-(C-\lambda A) X D-(F-\lambda E) M_{\lambda} D \\
& =E-A X \lambda D+\lambda A X D-(F-\lambda E) M_{\lambda} D-E-(\lambda E-F) M_{\lambda} D \\
& =0, \\
F+C Y+Z B & =F+C X(B-\lambda D)-(C-\lambda A) X B-(F-\lambda E) M_{\lambda} B \\
& =F+C X B-C X B-(F-\lambda E) M_{\lambda} B-\lambda\left(E+(\lambda E-F) M_{\lambda} D\right) \\
& =(F-\lambda E)-(F-\lambda E) M_{\lambda} B-\lambda(\lambda E-F) M_{\lambda} D \\
& =(F-\lambda E)\left(I_{q}-M_{\lambda}(B-\lambda D)\right) \\
& =0 .
\end{aligned}
$$

The following result is well known and since it considers only a regular matrix pencil we do not repeat its proof here. However, we need to introduce the notion of spectrum of a regular pencil $s E-A \in \mathbb{K}^{n \times n}[s]$ : this is the set of all $\lambda \in \mathbb{C} \cup\{\infty\}$ such that $\operatorname{rank}_{\mathbb{C}}(\lambda E-A)<n$.

LEMMA 4.15 (solvability of the generalized Sylvester equation: regular case [11, 17]). Let $A, C \in \mathbb{K}^{n \times n}, B, D \in \mathbb{K}^{p \times p}, E \in \mathbb{K}^{n \times p}$, and consider the generalized Sylvester equation (4.3). Assume that $(s B-D)$ and $(s C-A)$ are both regular with distinct spectra. Then (4.3) is solvable.

Finally, we can state and prove the result about the solvability of the generalized sylvester equation which is needed to prove Theorem 2.6.

LEmma 4.16 (solvability of the generalized Sylvester equation with special properties). Let $A, C \in \mathbb{K}^{m \times n}, m \leq n, B, D \in \mathbb{K}^{p \times q}, p>q, E \in \mathbb{K}^{m \times q}$, and consider the generalized Sylvester equation (4.3). Assume that $(\lambda B-D)$ has full rank for all $\lambda \in \mathbb{C} \cup\{\infty\}$ and that either $(\lambda C-A)$ has full rank for all $\lambda \in \mathbb{C} \cup\{\infty\}$ or that $(s C-A)$ is regular. Then (4.3) is solvable.

Proof. The proof follows that of [17, Thm. 2]. By Theorem 2.2 and Lemmas 4.12 and 4.13 we already know that we can put the pencils $s C-A$ and $s B-D$ into KCF. Therefore, choose invertible $S_{1}, T_{1}, S_{2}, T_{2}$ such that $s C_{0}-A_{0}=S_{1}(s C-A) T_{1}$ and $s B_{0}-D_{0}=S_{2}(s B-D) T_{2}$ are in KCF. Hence, with $X_{0}=T_{1}^{-1} X S_{1}^{-1}$ and $E_{0}=S_{1} E T_{2}$, equation (4.3) is equivalent to

$$
A_{0} X_{0} B_{0}-C_{0} X_{0} D_{0}=E_{0} .
$$


Let $s C_{0}-A_{0}=\operatorname{diag}\left(s C_{0}^{1}-A_{0}^{1}, \ldots, s C_{0}^{n_{1}}-A_{0}^{n_{1}}\right), n_{1} \in \mathbb{N}$, and $s B_{0}-D_{0}=\operatorname{diag}\left(s B_{0}^{1}-\right.$ $\left.D_{0}^{1}, \ldots, s B_{0}^{n_{2}}-D_{0}^{n_{2}}\right), n_{2} \in \mathbb{N}$, corresponding to the block structure of the KCF as in Corollary 2.8, then (4.3) is furthermore equivalent to the set of equations

$$
A_{0}^{i} X_{0}^{i j} B_{0}^{j}-C_{0}^{i} X_{0}^{i j} D_{0}^{j}=E_{0}^{i j}, \quad i=1, \ldots, n_{1}, j=1, \ldots, n_{2},
$$

where $X_{0}^{i j}$ and $E_{0}^{i j}$ are the corresponding subblocks of $X_{0}$ and $E_{0}$, respectively. Note that (using the notation of Corollary 2.8) by assumption each pencil $s C_{0}^{i}-A_{0}^{i}$ is a $\mathcal{P}_{\varepsilon}(s), \mathcal{J}_{\rho}(s)$, or $\mathcal{N}_{\sigma}(s)$ block, and all pencils $s B_{0}^{j}-D_{0}^{j}$ are $\mathcal{Q}_{\eta}(s)$ blocks. If $s C_{0}^{i}-A_{0}^{i}$ is a $\mathcal{P}_{\varepsilon}(s)$ block, we consider a reduced equation by deleting the first column of $s C_{0}^{i}-A_{0}^{i}$, which results in a regular $\mathcal{J}_{\varepsilon}(s)$ block with spectrum $\{0\}$, and by deleting the last row of $s B_{0}^{j}-D_{0}^{j}$, which results in a regular $\mathcal{N}_{\eta}(s)$ block with spectrum $\{\infty\}$. Hence, we use Lemma 4.15 to obtain solvability of the reduced problem. Filling the reduced solution for $X_{0}^{i j}$ by a zero row and a zero column results in a solution for the original problem. If $s C_{0}^{i}-A_{0}^{i}$ is a $\mathcal{J}_{\rho}(s)$ block, we can apply the same trick (this time we delete only the last row of $s B_{0}^{j}-D_{0}^{j}$ ) to arrive at the same conclusion, as $s C_{0}^{i}-A_{0}^{i}$ has only finite spectrum. Finally, if $s C_{0}^{i}-A_{0}^{i}$ is a $\mathcal{N}_{\sigma}(s)$ block with spectrum $\{\infty\}$, we have to reduce $s B_{0}^{j}-D_{0}^{j}$ by deleting the first row, which results in a $\mathcal{J}_{\eta}(s)$ block with spectrum $\{0\}$. This concludes the proof.

4.7. Solutions of DAEs. In order to prove Theorem 3.2 we need the following lemmas, which characterize the solutions of DAEs in the case of full rank pencils. As in section 3 we restrict ourselves to the case $\mathbb{K}=\mathbb{R}$.

Lemma 4.17 (full row rank pencils). Let $s E-A \in \mathbb{R}^{m \times n}[s]$ such that $m<n$ and $\operatorname{rank}_{\mathbb{C}}(\lambda E-A)=m$ for all $\lambda \in \mathbb{C} \cup\{\infty\}$. According to Lemma 3.1 choose $M(s) \in \mathbb{R}^{n \times m}[s]$ and $K(s) \in \mathbb{R}^{n \times(n-m)}[s]$ such that $(s E-A)[M(s), K(s)]=[I, 0]$

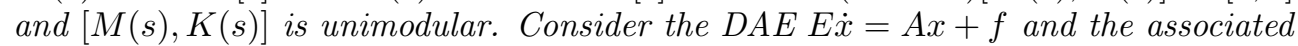
solution space $\mathcal{S}=\mathcal{C}^{\infty}$ or $\mathcal{S}=\mathbb{D}_{\text {pwC }} \infty$. Then, for all inhomogeneities $f \in \mathcal{S}^{m}, x \in \mathcal{S}^{n}$ is a solution if and only if there exists $u \in \mathcal{S}^{n-m}$ such that

$$
x=M\left(\frac{\mathrm{d}}{\mathrm{d} t}\right)(f)+K\left(\frac{\mathrm{d}}{\mathrm{d} t}\right)(u) .
$$

Furthermore, all initial value problems have a solution, i.e., for all $x^{0} \in \mathbb{R}^{n}, t_{0} \in \mathbb{R}$, and all $f \in \mathcal{S}^{m}$ there exists a solution $x \in \mathcal{S}^{n}$ such that

$$
x\left(t_{0}-\right)=x^{0} .
$$

Proof. Step 1. We show that $x=M\left(\frac{\mathrm{d}}{\mathrm{d} t}\right)(f)+K\left(\frac{\mathrm{d}}{\mathrm{d} t}\right)(u)$ solves $E \dot{x}=A x+f$ for any $u \in \mathcal{S}^{n-m}$.

This is clear since

$$
\left(E \frac{\mathrm{d}}{\mathrm{d} t}-A\right)\left(M\left(\frac{\mathrm{d}}{\mathrm{d} t}\right)(f)+K\left(\frac{\mathrm{d}}{\mathrm{d} t}\right)(u)\right)=f+0=f .
$$

Step 2 . We show that any solution $x$ of the DAE can be represented as above.

To this end let $u:=[0, I]\left[M\left(\frac{\mathrm{d}}{\mathrm{d} t}\right), K\left(\frac{\mathrm{d}}{\mathrm{d} t}\right)\right]^{-1} x \in \mathcal{S}^{n-m}$, which is well defined due to the unimodularity of $[M(s), K(s)]$. Then

$$
\begin{array}{r}
\left.f=\left(E \frac{\mathrm{d}}{\mathrm{d} t}-A\right) x=\left(E \frac{\mathrm{d}}{\mathrm{d} t}-A\right)\left[M\left(\frac{\mathrm{d}}{\mathrm{d} t}\right), K\left(\frac{\mathrm{d}}{\mathrm{d} t}\right)\right] \begin{array}{r}
{[I, 0]\left[M\left(\frac{\mathrm{d}}{\mathrm{d} t}\right), K\left(\frac{\mathrm{d}}{\mathrm{d} t}\right)\right]^{-1} x} \\
{[0, I]\left[M\left(\frac{\mathrm{d}}{\mathrm{d} t}\right), K\left(\frac{\mathrm{d}}{\mathrm{d} t}\right)\right]^{-1} x}
\end{array}\right] \\
=[I, 0]\left[M\left(\frac{\mathrm{d}}{\mathrm{d} t}\right), K\left(\frac{\mathrm{d}}{\mathrm{d} t}\right)\right]^{-1} x
\end{array}
$$


and therefore it follows that

$$
M\left(\frac{\mathrm{d}}{\mathrm{d} t}\right) f+K\left(\frac{\mathrm{d}}{\mathrm{d} t}\right) u=\left[M\left(\frac{\mathrm{d}}{\mathrm{d} t}\right), K\left(\frac{\mathrm{d}}{\mathrm{d} t}\right)\right]\left[\begin{array}{l}
{[I, 0]\left[M\left(\frac{\mathrm{d}}{\mathrm{d} t}\right), K\left(\frac{\mathrm{d}}{\mathrm{d} t}\right)\right]^{-1} x} \\
{[0, I]\left[M\left(\frac{\mathrm{d}}{\mathrm{d} t}\right), K\left(\frac{\mathrm{d}}{\mathrm{d} t}\right)\right]^{-1} x}
\end{array}\right]=x
$$

Step 3 . We show that every initial value is possible.

Write $K(s)=K_{0}+K_{1} s+\ldots+K_{p} s^{p}, p \in \mathbb{N}$, and let $\mathcal{K}$ be the singular chain manifold of $s E-A$ as in Corollary 4.8.

Step 3a. We show $\operatorname{im}\left[K_{0}, K_{1}, \ldots, K_{p}\right]=\mathcal{K}=\mathbb{R}^{n}$.

Remark 2.4 and Lemma 4.9 yield $\mathbb{R}^{n}=\mathcal{V}^{*} \cap \mathcal{W}^{*}=\mathcal{K}$. From $(s E-A) K(s)=0$ it follows that

$$
0=A K_{0}, E K_{0}=A K_{1}, \ldots, E K_{p-1}=A K_{p}, E K_{p}=\text {; }
$$

hence the $i$ th column vectors of $K_{0}, K_{1}, \ldots, K_{p}, i=1, \ldots, n-m$, form a singular chain. This shows $\operatorname{im}\left[K_{0}, K_{1}, \ldots, K_{p}\right] \subseteq \mathcal{K}$.

For showing the converse inclusion, we first prove im $K_{0}=\operatorname{ker} A$. From $A K_{0}=$ $\left.(\lambda E-A) K(\lambda)\right|_{\lambda=0}=0$ it follows that im $K_{0} \subseteq \operatorname{ker} A$. By unimodularity of $[M(s), K(s)]$ it follows that $K(0)=K_{0}$ must have full rank, i.e., $\operatorname{dim} \operatorname{im} K_{0}=n-m$. Full rank of $(s E-A)$ for all $s \in \mathbb{C}$ also implies full rank of $A$; hence $\operatorname{dim} \operatorname{ker} A=n-m$ and $\operatorname{im} K_{0}=\operatorname{ker} A$ is shown.

Let $\left(x_{0}, x_{1}, \ldots, x_{l}\right), l \in \mathbb{N}$, be a singular chain. Then $A x_{0}=0$, i.e., $x_{0} \in \operatorname{ker} A=$ $\operatorname{im} K_{0}$. Proceeding inductively, assume $x_{0}, x_{1}, \ldots, x_{i} \in \operatorname{im}\left[K_{0}, K_{1}, \ldots, K_{i}\right]$ for some $i \in \mathbb{N}$ with $0 \leq i<l$. For notational convenience set $K_{j}=0$ for all $j>p$. From $A x_{i+1}=E x_{i} \in \operatorname{im}\left[E K_{0}, E K_{1}, \ldots, E K_{i}\right]=\operatorname{im}\left[A K_{1}, A K_{2}, \ldots, A K_{i+1}\right]$ it follows that $x_{i+1} \in \operatorname{ker} A+\operatorname{im}\left[K_{1}, K_{2}, \ldots, K_{i+1}\right]=\operatorname{im}\left[K_{0}, K_{1}, \ldots, K_{i+1}\right]$. This shows that each singular chain is contained in $\operatorname{im}\left[K_{0}, K_{1}, \ldots, K_{p}\right]$.

Step $3 \mathrm{~b}$. We show existence of $u \in \mathcal{S}^{n-m}$ such that $x\left(t_{0}-\right)=x^{0}$.

By Step 3a there exist $u_{0}, u_{1}, \ldots, u_{p} \in \mathbb{R}^{n-m}$ such that

$$
K_{0} u_{0}+K_{1} u_{1}+\cdots+K_{p} u_{p}=x^{0}-M\left(\frac{\mathrm{d}}{\mathrm{d} t}\right)(f)\left(t_{0}-\right) .
$$

Let

$$
u(t):=u_{0}+\left(t-t_{0}\right) u_{1}+\frac{\left(t-t_{0}\right)^{2}}{2} u_{2}+\cdots+\frac{\left(t-t_{0}\right)^{p}}{p !} u_{p}, \quad t \in \mathbb{R} .
$$

Then we have that $u \in \mathcal{S}$ and

$$
K\left(\frac{\mathrm{d}}{\mathrm{d} t}\right)(u)\left(t_{0}-\right)=K_{0} u_{0}+K_{1} u_{1}+\cdots+K_{p} u_{p} \stackrel{(4.5)}{=} x^{0}-M\left(\frac{\mathrm{d}}{\mathrm{d} t}\right)(f)\left(t_{0}-\right),
$$

which implies that the solution $x=M\left(\frac{\mathrm{d}}{\mathrm{d} t}\right)(f)+K\left(\frac{\mathrm{d}}{\mathrm{d} t}\right)(u)$ satisfies

$$
x\left(t_{0}-\right)=M\left(\frac{\mathrm{d}}{\mathrm{d} t}\right)(f)\left(t_{0}-\right)+K\left(\frac{\mathrm{d}}{\mathrm{d} t}\right)(u)\left(t_{0}-\right) \stackrel{(4.6)}{=} x^{0} .
$$

Remark 4.18. A careful analysis of the proof of Lemma 4.17 reveals that for the solution formula the full rank of $\lambda E-A$ for $\lambda=\infty$ is not necessary. The latter is only necessary to show that all initial value problems have a solution.

LEMma 4.19 (full column rank pencils). Let $s E-A \in \mathbb{R}^{m \times n}[s]$ such that $m>n$ and $\operatorname{rank}_{\mathbb{C}}(\lambda E-A)=n$ for all $\lambda \in \mathbb{C} \cup\{\infty\}$. According to Lemma 3.1 choose 
$M(s) \in \mathbb{R}^{n \times m}[s]$ and $K(s) \in \mathbb{R}^{(m-n) \times m}[s]$ such that $\left[\begin{array}{c}M(s) \\ K(s)\end{array}\right](s E-A)=\left[\begin{array}{l}I \\ 0\end{array}\right]$ and $\left[\begin{array}{c}M(s) \\ K(s)\end{array}\right]$ is unimodular. Then, for $f \in \mathcal{S}^{m}, x \in \mathcal{S}^{n}$ is a solution of $E \dot{x}=A x+f$ if and only if

$$
x=M\left(\frac{\mathrm{d}}{\mathrm{d} t}\right)(f) \quad \text { and } \quad K\left(\frac{\mathrm{d}}{\mathrm{d} t}\right)(f)=0 .
$$

Furthermore, every component or linear combination of $f$ is restricted in some way; more precisely $K(s) F$ has no zero column for any invertible $F \in \mathbb{R}^{m \times m}$.

Proof. The characterization of the solution follows from the equivalence

$$
\left(E \frac{\mathrm{d}}{\mathrm{d} t}-A\right) x=f \Longleftrightarrow \underbrace{\left[\begin{array}{c}
M\left(\frac{\mathrm{d}}{\mathrm{d} t}\right) \\
K\left(\frac{\mathrm{d}}{\mathrm{d} t}\right)
\end{array}\right]\left(E \frac{\mathrm{d}}{\mathrm{d} t}-A\right)}_{=\left[\begin{array}{l}
I \\
0
\end{array}\right]} x=\left[\begin{array}{c}
M\left(\frac{\mathrm{d}}{\mathrm{d} t}\right) f \\
K\left(\frac{\mathrm{d}}{\mathrm{d} t}\right) f
\end{array}\right] .
$$

To show that $K(s) F$ does not have any zero column, write $K(s)=K_{0}+K_{1} s+\cdots+$ $K_{k} s^{k}$. Since $\left(s E^{\top}-A^{\top}\right) K(s)^{\top}=0$ it follows with the same arguments as in Step 3a of Lemma 4.17 that $\operatorname{im}\left[K_{0}^{\top}, K_{1}^{\top}, \ldots, K_{k}^{\top}\right]=\mathbb{R}^{m}$. Hence, $\operatorname{ker}\left[K_{0}^{\top}, K_{1}^{\top}, \ldots, K_{k}^{\top}\right]^{\top}=\{0\}$, which shows that the only $v \in \mathbb{R}^{m}$ with $K_{i} v=0$ for all $i=1, \ldots, k$ is $v=0$. This shows that $K(s) F$ does not have a zero column for any invertible $F \in \mathbb{R}^{m \times m}$.

Remark 4.20. Analogously, as pointed out in Remark 4.18, the condition that $\lambda E-A$ must have full rank for $\lambda=\infty$ is not needed to characterize the solution. It is only needed to show that the inhomogeneity is "completely" restricted.

\section{Proofs of the main results.}

5.1. Proof of Theorem 2.3: The QKTF. We are now ready to prove our main result about the QKTF. We proceed in several steps.

Step 1. We show the block-triangular form (2.3).

By the choice of $P_{1}, R_{1}, Q_{1}$ and $P_{2}, R_{2}, Q_{2}$ it follows immediately that $T_{\text {trian }}$ and $S_{\text {trian }}$ are invertible. Note that (2.3) is equivalent to the solvability (for given $E, A$, and $P_{1}, R_{1}, Q_{1}, P_{2}, R_{2}, Q_{2}$ ) of

$$
\begin{array}{ll}
E P_{1}=P_{2} E_{P}, & A P_{1}=P_{2} A_{P}, \\
E R_{1}=P_{2} E_{P R}+R_{2} E_{R}, & A R_{1}=P_{2} A_{P R}+R_{2} A_{R}, \\
E Q_{1}=P_{2} E_{P Q}+R_{2} E_{R Q}+Q_{2} E_{Q}, & A Q_{1}=P_{2} A_{P Q}+R_{2} A_{R Q}+Q_{2} A_{Q} .
\end{array}
$$

The solvability of the latter is implied by the following subspace inclusions:

$$
\begin{aligned}
& E\left(\mathcal{V}^{*} \cap \mathcal{W}^{*}\right) \subseteq E \mathcal{V}^{*} \cap A \mathcal{W}^{*}, \quad A\left(\mathcal{V}^{*} \cap \mathcal{W}^{*}\right) \subseteq E \mathcal{V}^{*} \cap A \mathcal{W}^{*}, \\
& E\left(\mathcal{V}^{*}+\mathcal{W}^{*}\right) \subseteq E \mathcal{V}^{*}+A \mathcal{W}^{*}, \quad A\left(\mathcal{V}^{*}+\mathcal{W}^{*}\right) \subseteq E \mathcal{V}^{*}+A \mathcal{W}^{*}, \\
& E \mathbb{K}^{n} \subseteq \mathbb{K}^{m}, \quad A \mathbb{K}^{n} \subseteq \mathbb{K}^{m},
\end{aligned}
$$

which clearly hold due to (2.2).

Step 2. We show (i).

Step 2a. Full row rank of $E_{P}$ and $A_{P}$.

From Lemma 4.4 it follows that

$$
\operatorname{im} P_{2} E_{P}=\operatorname{im} E P_{1}=\operatorname{im} P_{2} \quad \text { and } \quad \operatorname{im} P_{2} A_{P}=\operatorname{im} A P_{1}=\operatorname{im} P_{2} ;
$$

hence, invoking the full column rank of $P_{2}$, im $E_{P}=\mathbb{K}^{m_{P}}=\operatorname{im} A_{P}$, which implies full row rank of $E_{P}$ and $A_{P}$. In particular this shows full row rank of $\lambda E_{P}-A_{P}$ for $\lambda=0$ and $\lambda=\infty$. 
Step 2b. Full row rank of $\lambda E_{P}-A_{P}$ for all $\lambda \in \mathbb{C} \backslash\{0\}$.

Seeking a contradiction, assume existence of $\lambda \in \mathbb{C} \backslash\{0\}$ with $\operatorname{rank}_{\mathbb{C}}\left(\lambda E_{P}-A_{P}\right)<$ $m_{P}$. Then there exists $v \in \mathbb{C}^{m_{P}}$ such that $v^{\top}\left(\lambda E_{P}-A_{P}\right)=0$. Full column rank of $P_{2} \in \mathbb{K}^{m \times m_{P}}$ implies existence of $w \in \mathbb{C}^{m}$ such that $w^{\top} P_{2}=v^{\top}$; hence

$$
0=v^{\top}\left(\lambda E_{P}-A_{P}\right)=w^{\top}\left(\lambda P_{2} E_{P}-P_{2} A_{P}\right)=w^{\top}(\lambda E-A) P_{1} .
$$

Invoking Lemma 4.9 , there exists a linearly independent singular chain $\left(x_{0}, x_{1}, \ldots, x_{k}\right)$ such that

$$
\operatorname{span}\left\{x_{0}, x_{1}, \ldots, x_{k}\right\}=\operatorname{im} P_{1}=\mathcal{V}^{*} \cap \mathcal{W}^{*} .
$$

In particular, $x_{i} \in \operatorname{im} P_{1}$ for $i=0,1, \ldots$, implies

$$
\forall i \in\{0,1, \ldots, k\}: \quad w^{\top}(\lambda E-A) x_{i}=0 .
$$

Since $E x_{k}=0$ it follows that $w^{\top} A x_{k}=0$ and inductively it follows that

$$
0=w^{\top}\left(\lambda E x_{i-1}-A x_{i-1}\right)=w^{\top}\left(\lambda A x_{i}-A x_{i-1}\right)=-w^{\top} A x_{i-1}
$$

and, therefore,

$$
0=w^{\top} A P_{1}=w^{\top} P_{2} A_{P}=v^{\top} A_{P} .
$$

This shows that $A_{P} \in \mathbb{K}^{m_{P} \times n_{P}}$ does not have full row rank over $\mathbb{C}$ which implies also a row rank defect over $\mathbb{K}$. This is the sought contradiction because the full row rank of $A_{P}$ was already shown in Step 2a.

Step 3. We show (ii).

For notational convenience let $\mathcal{K}:=\mathcal{V}^{*} \cap \mathcal{W}^{*}$.

Step 3a. We show that $m_{R}=n_{R}$.

Invoking

$$
\operatorname{ker} E \cap \mathcal{K}=\operatorname{ker} E \cap \mathcal{V}^{*}, \quad \operatorname{ker} A \cap \mathcal{K}=\operatorname{ker} A \cap \mathcal{W}^{*},
$$

and Lemmas 4.3 and 4.4, the claim follows from

$$
\begin{aligned}
m_{R}= & \operatorname{rank} R_{2} \\
= & \operatorname{dim}\left(E \mathcal{V}^{*}+A \mathcal{W}^{*}\right)-\operatorname{dim}\left(E \mathcal{V}^{*} \cap A \mathcal{W}^{*}\right) \\
= & \operatorname{dim} E \mathcal{V}^{*}+\operatorname{dim} A \mathcal{W}^{*}-2 \operatorname{dim}\left(E \mathcal{V}^{*} \cap A \mathcal{W}^{*}\right) \\
= & \operatorname{dim} \mathcal{V}^{*}-\operatorname{dim}\left(\operatorname{ker} E \cap \mathcal{V}^{*}\right)+\operatorname{dim} \mathcal{W}^{*}-\operatorname{dim}\left(\operatorname{ker} A \cap \mathcal{W}^{*}\right)-\operatorname{dim} E \mathcal{K}-\operatorname{dim} A \mathcal{K} \\
= & \operatorname{dim} \mathcal{V}^{*}-\operatorname{dim}\left(\operatorname{ker} E \cap \mathcal{V}^{*}\right)+\operatorname{dim} \mathcal{W}^{*}-\operatorname{dim}\left(\operatorname{ker} A \cap \mathcal{W}^{*}\right)-\operatorname{dim} \mathcal{K} \\
& +\operatorname{dim}(\operatorname{ker} E \cap \mathcal{K})-\operatorname{dim} \mathcal{K}+\operatorname{dim}(\operatorname{ker} A \cap \mathcal{K}) \\
& \stackrel{(5.1)}{=} \operatorname{dim} \mathcal{V}^{*}+\operatorname{dim} \mathcal{W}^{*}-2 \operatorname{dim} \mathcal{K} \\
= & \operatorname{dim}\left(\mathcal{V}^{*}+\mathcal{W}^{*}\right)-\operatorname{dim}\left(\mathcal{V}^{*} \cap \mathcal{W}^{*}\right) \\
= & \operatorname{rank} R_{1}=n_{R} .
\end{aligned}
$$

Step 3b. We show that $\operatorname{det}\left(s E_{R}-A_{R}\right) \not \equiv 0$.

Seeking a contradiction, assume $\operatorname{det}\left(s E_{R}-A_{R}\right)$ is the zero polynomial. Then $\lambda E_{R}-A_{R}$ has a column rank defect for all $\lambda \in \mathbb{C} \cup\{\infty\}$; hence

$$
\forall \lambda \in \mathbb{C} \cup\{\infty\}: \operatorname{rank}_{\mathbb{C}}\left(\lambda E_{R}-A_{R}\right)<n_{R}
$$


Now, Lemma 4.10 ensures existence of a nontrivial singular chain $\left(y_{0}, y_{1}, \ldots, y_{k}\right)$ of the matrix pencil $s E_{R}-A_{R}$.

We show that there exists a singular chain $\left(x_{0}, x_{1}, \ldots, x_{k}, x_{k+1}, \ldots, x_{\hat{k}}\right)$ of $s E-A$ such that $x_{i}=\left[P_{1}, R_{1}\right]\left(\begin{array}{c}z_{i} \\ y_{i}\end{array}\right)$ for $i=0, \ldots, k$. To this end denote some right inverse of $A_{P}$ (invoking full row rank of $A_{P}$ as shown in Step 2a) with $A_{P}^{+}$and let

$$
z_{0}=-A_{P}^{+} A_{P R} y_{0}, \quad z_{i+1}=A_{P}^{+}\left(E_{P} z_{i}+E_{P R} y_{i}-A_{P R} y_{i+1}\right), \quad i=0, \ldots, k,
$$

where $y_{k+1}=0$. Then it follows that

$$
\begin{aligned}
A x_{i} & =A\left[P_{1}, R_{1}\right]\left(\begin{array}{c}
z_{i} \\
y_{i}
\end{array}\right)=A T_{\text {trian }}\left(\begin{array}{c}
z_{i} \\
y_{i} \\
0
\end{array}\right)=S_{\text {trian }}^{-1}\left[\begin{array}{ccc}
A_{P} & A_{P R} & A_{P Q} \\
0 & A_{R} & A_{R Q} \\
0 & 0 & A_{Q}
\end{array}\right]\left(\begin{array}{c}
z_{i} \\
y_{i} \\
0
\end{array}\right) \\
& =S_{\text {trian }}^{-1}\left(\begin{array}{c}
A_{P} z_{i}+A_{P R} y_{i} \\
A_{R} y_{i} \\
0
\end{array}\right)
\end{aligned}
$$

and, analogously,

$$
E x_{i}=S_{\text {trian }}^{-1}\left(\begin{array}{c}
E_{P} z_{i}+E_{P R} y_{i} \\
E_{R} y_{i} \\
0
\end{array}\right)
$$

hence $A x_{0}=0$ and $E x_{i}=A x_{i+1}$ for $i=0, \ldots, k$. Note that, if we set $x_{k+1}=P_{1} z_{k+1}$, then $x_{k+1} \in \mathcal{V}^{*} \cap \mathcal{W}^{*} \subseteq \mathcal{W}^{*}$, and identically as shown in the first part of Step 2 of the proof of Lemma 4.9 there exist $x_{k+2}, \ldots, x_{\hat{k}}, \hat{k}>k$ such that $E x_{k+1}=$ $A x_{k+2}, \ldots, E x_{\hat{k}-1}=A x_{\hat{k}}, E x_{\hat{k}}=0$ and, therefore, $\left(x_{0}, x_{1}, \ldots, x_{\hat{k}}\right)$ is a singular chain of $s E-A$. Lemma 4.9 implies that $\left\{x_{0}, x_{1}, \ldots, x_{\hat{k}}\right\} \subseteq \operatorname{im} P_{1}$; hence $x_{i}=\left[P_{1}, R_{1}\right]\left(\begin{array}{c}z_{i} \\ y_{i}\end{array}\right)$ implies $y_{i}=0$ for all $i \in\{0, \ldots, k\}$, which contradicts nontriviality of $\left(y_{0}, \ldots, y_{k}\right)$.

Step 4 . We show (iii).

We will consider the transposed matrix pencil $s E^{\top}-A^{\top}$ with corresponding Wong sequences and show that the block $\left(E_{Q}^{\top}, A_{Q}^{\top}\right)$ will play the role of the block $\left(E_{P}, A_{P}\right)$. Therefore, denote the limits of the Wong sequences of $s E^{\top}-A^{\top}$ by $\widehat{\mathcal{V}}^{*}$ and $\widehat{\mathcal{W}}^{*}$. Let

$$
\widehat{Q}_{1}:=\left(\left[0,0, I_{n_{Q}}\right]\left[P_{1}, R_{1}, Q_{1}\right]^{-1}\right)^{\top} \quad \text { and } \quad \widehat{Q}_{2}:=\left(\left[0,0, I_{m_{Q}}\right]\left[P_{2}, R_{2}, Q_{2}\right]^{-1}\right)^{\top} ;
$$

then

$$
\widehat{Q}_{i}^{\top} Q_{i}=I \quad \text { and } \quad \operatorname{im} \widehat{Q}_{i}=\left(\operatorname{im}\left[P_{i}, R_{i}\right]\right)^{\perp} \quad \text { for } i=1,2 .
$$

In fact, the latter follows from $n-n_{Q}=n_{P}+n_{R}$ and

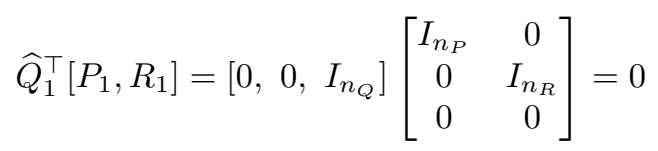

for $i=1$ and analogously for $i=2$. We will show in the following that

$$
\begin{array}{ll}
E^{\top} \widehat{Q}_{2}=\widehat{Q}_{1} E_{Q}^{\top}, & A^{\top} \widehat{Q}_{2}=\widehat{Q}_{1} A_{Q}^{\top}, \\
\operatorname{im} \widehat{Q}_{2}=\widehat{\mathcal{V}}^{*} \cap \widehat{\mathcal{W}}^{*}, & \operatorname{im} \widehat{Q}_{1}=E^{\top} \widehat{\mathcal{V}}^{*} \cap A^{\top} \widehat{\mathcal{V}}^{*} ;
\end{array}
$$

then the arguments from Step 2 can be applied to $s E_{Q}^{\top}-A_{Q}^{\top}$ and the claim is shown. 
Step 4a. We show $E^{\top} \widehat{Q}_{2}=\widehat{Q}_{1} E_{Q}^{\top}$ and $A^{\top} \widehat{Q}_{2}=\widehat{Q}_{1} A_{Q}^{\top}$.

Using (2.3) we obtain

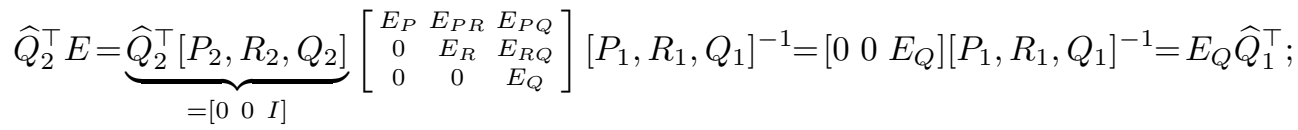

hence $E^{\top} \widehat{Q}_{2}=\widehat{Q}_{1} E_{Q}^{\top}$. Analogous arguments show that $A^{\top} \widehat{Q}_{2}=\widehat{Q}_{1} A_{Q}^{\top}$.

Step $4 \mathrm{~b}$. We show $\operatorname{im} \widehat{Q}_{2}=\widehat{\mathcal{V}}^{*} \cap \widehat{\mathcal{W}}^{*}$.

By construction and Lemma 4.5,

$\operatorname{im} \widehat{Q}_{2}=\left(\operatorname{im}\left[P_{2}, R_{2}\right]\right)^{\perp}=\left(E \mathcal{V}^{*}+A \mathcal{W}^{*}\right)^{\perp}=\left(E \mathcal{V}^{*}\right)^{\perp} \cap\left(A \mathcal{W}^{*}\right)^{\perp}=\widehat{\mathcal{V}}^{*} \cap \widehat{\mathcal{W}}^{*}$

Step 4c. We show $\operatorname{im} \widehat{Q}_{1}=E^{\top} \widehat{\mathcal{V}}^{*} \cap A^{\top} \widehat{\mathcal{V}}^{*}$.

Lemma 4.5 applied to $\left(E^{\top}, A^{\top}\right)$ gives

$$
\left(E^{\top} \widehat{\mathcal{V}}^{*}\right)^{\perp}=\mathcal{W}^{*} \quad \text { and } \quad\left(A^{\top} \widehat{\mathcal{W}}^{*}\right)^{\perp}=\mathcal{V}^{*}
$$

or, equivalently,

$$
E^{\top} \widehat{\mathcal{V}}^{*}=\mathcal{W}^{* \perp} \text { and } A^{\top} \widehat{\mathcal{W}}^{*}=\mathcal{V}^{* \perp}
$$

Hence

$$
\operatorname{im} \widehat{Q}_{1}=\left(\operatorname{im}\left[P_{1}, R_{1}\right]\right)^{\perp}=\left(\mathcal{V}^{*}+\mathcal{W}^{*}\right)^{\perp}=\mathcal{V}^{* \perp} \cap \mathcal{W}^{* \perp}=A^{\top} \widehat{\mathcal{W}}^{*} \cap E^{\top} \widehat{\mathcal{V}}^{*} .
$$

This concludes the proof of our first main result.

5.2. Proof of Theorem 2.6: The QKF. By the properties of the pencils $s E_{P}-A_{P}, s E_{R}-A_{R}$ and $s E_{Q}-A_{Q}$ there exist $\lambda \in \mathbb{K}$ and full rank matrices $N_{\lambda}^{P}, N_{\lambda}^{R}$, $M_{\lambda}^{R}$, and $M_{\lambda}^{Q}$ such that $\left(\lambda E_{P}-A_{P}\right) N_{\lambda}^{P}=I,\left(\lambda E_{R}-A_{R}\right) N_{\lambda}^{R}=I, M_{\lambda}^{R}\left(\lambda E_{R}-A_{R}\right)=I$, and $M_{\lambda}^{Q}\left(\lambda E_{Q}-A_{Q}\right)=I$. Hence Lemma 4.14 shows that it suffices to consider solvability of the following generalized Sylvester equations:

$$
\begin{aligned}
E_{R} X_{1} A_{Q}-A_{R} X_{1} E_{Q}= & -E_{R Q}-\left(\lambda E_{R Q}-A_{R Q}\right) M_{\lambda}^{Q} E_{Q} \\
E_{P} X_{2} A_{R}-A_{P} X_{2} E_{R}= & -E_{P R}-\left(\lambda E_{P R}-A_{P R}\right) M_{\lambda}^{R} E_{R} \\
E_{P} X_{3} A_{Q}-A_{P} X_{3} E_{Q}= & -\left(E_{P Q}+E_{P R} F_{1}\right) \\
& -\left(\lambda\left(E_{P Q}+E_{P R} F_{1}\right)-\left(A_{P Q}+A_{P R} F_{1}\right)\right) M_{\lambda}^{Q} E_{Q},
\end{aligned}
$$

where $F_{1}$ is any solution of (2.4a), whose existence will follow from solvability of (5.2a). Furthermore, the properties of $s E_{P}-A_{P}, s E_{Q}-A_{Q}$, and $s E_{R}-A_{R}$ imply that Lemma 4.16 is applicable to (5.2) (where (5.2b) must be considered in the (conjugate) transposed form) and ensures existence of solutions. Now, a simple calculation shows that for any solution of (2.4) the second part of the statement of Theorem 2.6 holds.

Finally, to show uniqueness in the sense of $(2.6)$ assume first that $(E, A) \cong$ $\left(E^{\prime}, A^{\prime}\right)$. Then there exist invertible matrices $S^{\prime}$ and $T^{\prime}$ such that $\left(E^{\prime}, A^{\prime}\right)=\left(S^{\prime} E T^{\prime}\right.$, $\left.S^{\prime} A T^{\prime}\right)$. It is easily seen that the Wong sequences $\mathcal{V}_{i}^{\prime}, \mathcal{W}_{i}^{\prime}, i \in \mathbb{N}$, of the pencil $s E^{\prime}-A^{\prime}$ fulfill

$$
\mathcal{V}_{i}^{\prime}=T^{\prime-1} \mathcal{V}_{i}, \quad \mathcal{W}_{i}^{\prime}=T^{\prime-1} \mathcal{W}_{i}, \quad E^{\prime} \mathcal{V}_{i}^{\prime}=S^{\prime} E \mathcal{V}_{i}, \quad A^{\prime} \mathcal{W}_{i}^{\prime}=S^{\prime} A \mathcal{W}_{i}
$$


Hence, using the notation of Theorem 2.3, there exist invertible matrices $M_{P}, M_{R}$, $M_{Q}, N_{P}, N_{R}, N_{Q}$ such that

$$
\left[P_{1}^{\prime}, R_{1}^{\prime}, Q_{1}^{\prime}\right]=T^{\prime-1}\left[P_{1} M_{P}, R_{1} M_{R}, Q_{1} M_{Q}\right], \quad\left[P_{2}^{\prime}, R_{2}^{\prime}, Q_{2}^{\prime}\right]=S^{\prime}\left[P_{2} N_{P}, R_{2} N_{R}, Q_{2} N_{Q}\right] ;
$$

in particular the block sizes of the corresponding QKFs coincide. Therefore,

$$
\begin{aligned}
& s\left[\begin{array}{ccc}
E_{P} & * & * \\
0 & E_{R} & * \\
0 & 0 & E_{Q}
\end{array}\right]-\left[\begin{array}{ccc}
A_{P} & * & * \\
0 & A_{R} & * \\
0 & 0 & A_{Q}
\end{array}\right]=\left[P_{2}, R_{2}, Q_{2}\right]^{-1}(s E-A)\left[P_{1}, R_{1}, Q_{1}\right] \\
= & {\left[\begin{array}{ccc}
N_{P} & 0 & 0 \\
0 & N_{R} & 0 \\
0 & 0 & N_{Q}
\end{array}\right]\left[P_{2}^{\prime}, R_{2}^{\prime}, Q_{2}^{\prime}\right] S^{\prime}(s E-A) T^{\prime}\left[P_{1}^{\prime}, R_{2}^{\prime}, Q_{2}^{\prime}\right]\left[\begin{array}{ccc}
M_{P} & 0 & 0 \\
0 & M_{R} & 0 \\
0 & 0 & M_{Q}
\end{array}\right] } \\
= & {\left[\begin{array}{ccc}
N_{P} & 0 & 0 \\
0 & N_{R} & 0 \\
0 & 0 & N_{Q}
\end{array}\right]\left[P_{2}^{\prime}, R_{2}^{\prime}, Q_{2}^{\prime}\right]\left(s E^{\prime}-A^{\prime}\right)\left[P_{1}^{\prime}, R_{2}^{\prime}, Q_{2}^{\prime}\right]\left[\begin{array}{ccc}
M_{P}^{-1} & 0 & 0 \\
0 & M_{R}^{-1} & 0 \\
0 & 0 & M_{Q}^{-1}
\end{array}\right] } \\
= & s\left[\begin{array}{ccc}
N_{P} E_{P}^{\prime} M_{P}^{-1} & * & * \\
0 & N_{R} E_{R}^{\prime} M_{R}^{-1} & * \\
0 & 0 & N_{Q} E_{Q}^{\prime} M_{Q}^{-1}
\end{array}\right] \\
& -\left[\begin{array}{ccc}
N_{P} A_{P}^{\prime} M_{P}^{-1} & * & * \\
0 & N_{R} A_{R} M_{R}^{-1} & * \\
0 & 0 & N_{Q} A_{Q} M_{Q}^{-1}
\end{array}\right] .
\end{aligned}
$$

Hence the necessity part of (2.6) is shown. Sufficiency follows from the simple observation that equivalence of the QKFs implies equivalence of the original matrix pencils.

5.3. Proof of Theorem 2.9: The minimal indices. First consider two equivalent matrix pencils $s E-A$ and $s E^{\prime}-A^{\prime}$ and the relationship of the corresponding Wong sequences (5.3). From this it follows that the subspace dimension used for the calculations of $a, d, \Delta_{i}$ and $\widehat{\Delta}_{i}, i=0,1,2, \ldots$, are invariant under equivalence transformations. Hence it suffices to show the claim when the matrix pair is already in $\mathrm{KCF}$ as in Corollary 2.8. In particular, uniqueness of the KCF then also follows from this invariance.

Note that the Wong sequences of a matrix pencil in QKF (2.5) fulfill

$$
\mathcal{V}_{i}=\left[\begin{array}{l}
I \\
0 \\
0
\end{array}\right] \mathcal{V}_{i}^{P} \oplus\left[\begin{array}{l}
0 \\
I \\
0
\end{array}\right] \mathcal{V}_{i}^{R} \oplus\left[\begin{array}{l}
0 \\
0 \\
I
\end{array}\right] \mathcal{V}_{i}^{Q}, \quad \mathcal{W}_{i}=\left[\begin{array}{l}
I \\
0 \\
0
\end{array}\right] \mathcal{W}_{i}^{P} \oplus\left[\begin{array}{l}
0 \\
I \\
0
\end{array}\right] \mathcal{W}_{i}^{R} \oplus\left[\begin{array}{l}
0 \\
0 \\
I
\end{array}\right] \mathcal{W}_{i}^{Q},
$$

where $\mathcal{V}_{i}^{P}, \mathcal{W}_{i}^{P}, \mathcal{V}_{i}^{R}, \mathcal{W}_{i}^{R}, \mathcal{V}_{i}^{Q}, \mathcal{W}_{i}^{Q}, i \in \mathbb{N}$, are the Wong sequences corresponding to the matrix pencils $s E_{P}-A_{P}, s E_{R}-A_{R}$, and $s E_{Q}-A_{Q}$. Furthermore, due to Theorem 2.6 and the uniqueness result therein, it follows that

$$
\mathcal{V}^{*} \cap \mathcal{W}^{*}=\operatorname{im}\left[\begin{array}{lll}
I & 0 & 0 \\
0 & 0 & 0 \\
0 & 0 & 0
\end{array}\right]
$$

Altogether it is, therefore, sufficient to show the claim for the singular block $s E_{P}-A_{P}$ with its Wong sequences $\mathcal{V}_{i}^{P}$ and $\mathcal{W}_{i}^{P}$. The result for the singular block 
$s E_{Q}-A_{Q}$ follows by considering the (conjugate) transpose and invoking Lemma 4.5. Since $\mathcal{K}^{P}:=\mathcal{V}^{P^{*}} \cap \mathcal{W}^{P^{*}}=\mathbb{R}^{n_{p}}$, we have $\mathcal{W}_{i}^{P} \cap \mathcal{K}^{P}=\mathcal{W}_{i}^{P}$, and the claim simplifies again.

The remaining proof is quite simple but the notation is rather cumbersome. Therefore we accompany the proof with an illustrative example:

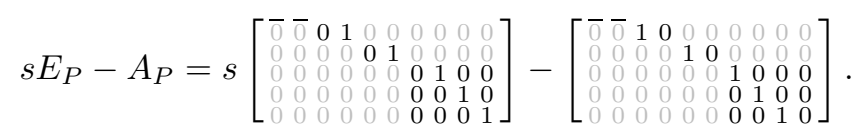

In this example $a=5, \varepsilon_{1}=\varepsilon_{2}=0, \varepsilon_{3}=\varepsilon_{4}=1$, and $\varepsilon_{5}=3$. Denote with $\kappa_{1}, \kappa_{2}, \ldots, \kappa_{a}$ the position where a new $P_{\varepsilon}(s)$ block begins in $s E_{P}-A_{P}$; for the example this is $\kappa_{1}=1, \kappa_{2}=2, \kappa_{3}=3, \kappa_{4}=5, \kappa_{5}=7$. By definition

$$
\mathcal{W}_{1}^{P}=\operatorname{ker} E_{P}=\operatorname{im}\left[e_{\kappa_{1}}, e_{\kappa_{2}}, \ldots, e_{\kappa_{a}}\right]=: \operatorname{im} W_{1}^{P},
$$

where $e_{\ell}$ denotes the $\ell$ th unit vector. This shows $a=\operatorname{dim} \mathcal{W}_{1}^{P}=\operatorname{dim}\left(\mathcal{W}_{1} \cap \mathcal{K}\right)$.

Let $\nu_{i}, i=0,1,2, \ldots, m_{P}$ be the number of $P_{\varepsilon}(s)$-blocks of size $i$ in $s E_{P}-A_{P}$; for our example (5.4) this means $\nu_{0}=2, \nu_{1}=2, \nu_{2}=0, \nu_{3}=1, \nu_{4}=0, \nu_{5}=0$. Then

$$
\begin{aligned}
A_{P} W_{1}^{P}= & {[\underbrace{0, \ldots, 0}_{\nu_{0}}, \underbrace{e_{1}, \ldots, e_{\nu_{1}}}_{\nu_{1}}, \underbrace{e_{\nu_{1}+1}, e_{\nu_{1}+3}, \ldots, e_{\nu_{1}+2\left(\nu_{2}-1\right)}}_{\nu_{2}}, \ldots,} \\
& \underbrace{e_{\nu_{1}+2 \nu_{2}+\cdots+\left(m_{P}-1\right) \nu_{m_{P}-1}+1}, \ldots, e_{\nu_{1}+2 \nu_{2}+\cdots+\left(m_{P}-1\right) \nu_{m_{P}-1}+m_{P}\left(\nu_{m_{P}}-1\right)}}_{\nu_{m_{P}}}] .
\end{aligned}
$$

For (5.4) this reads as

$$
A_{P} W_{1}^{P}=\left[\begin{array}{lllll}
0 & 0 & 1 & 0 & 0 \\
0 & 0 & 0 & 1 & 0 \\
0 & 0 & 0 & 0 & 1 \\
0 & 0 & 0 & 0 & 0 \\
0 & 0 & 0 & 0 & 0
\end{array}\right] .
$$

Denote with $\beta_{1}$ the smallest index such that $\varepsilon_{\beta_{1}} \geq 1$, if it exists, $\beta_{1}=a+1$ otherwise (for our example, $\beta_{1}=3$ ). It then follows that

$$
\begin{aligned}
\mathcal{W}_{2}^{P} & =E_{P}^{-1}\left(A_{P} \mathcal{W}_{1}^{P}\right) \\
& =\mathcal{W}_{1}^{P} \oplus \operatorname{im}\left[e_{\kappa_{\beta_{1}}+1}, e_{\kappa_{\beta_{1}+1}+1}, \ldots, e_{\kappa_{a}+1}\right] \\
& =\operatorname{im}\left[e_{\kappa_{1}}, \ldots, e_{\kappa_{\beta_{1}-1}}, e_{\kappa_{\beta_{1}}}, e_{\kappa_{\beta_{1}}+1}, e_{\kappa_{\beta_{1}+1}}, e_{\kappa_{\beta_{1}+1}+1}, \ldots, e_{\kappa_{a}}, e_{\kappa_{a}+1}\right] \\
& =: \operatorname{im} W_{2}^{P}
\end{aligned}
$$

For the example (5.4) this is

$$
\mathcal{W}_{2}^{P}=\mathcal{W}_{1}^{P} \oplus \operatorname{im}\left[e_{4}, e_{6}, e_{8}\right]=\operatorname{im}\left[e_{1}, e_{2}, e_{3}, e_{4}, e_{5}, e_{6}, e_{7}, e_{8}\right] .
$$

Since $\Delta_{0}=\operatorname{dim} \mathcal{W}_{2}^{P}-\operatorname{dim} \mathcal{W}_{1}^{P}=a-\left(\beta_{1}-1\right)$ and $\varepsilon_{1}=\cdots=\varepsilon_{\beta_{1}-1}=0$ we have shown that $\varepsilon_{1}=\cdots=\varepsilon_{a-\Delta_{0}}=0$.

With $\beta_{i}$ being the smallest index such that $\varepsilon_{\beta_{i}} \geq i$ (or $\beta_{i}=a+1$ if it does not exist) and with an analogue argument as above we inductively conclude that

$$
\mathcal{W}_{i+1}^{P}=\mathcal{W}_{i}^{P} \oplus \operatorname{im}\left[e_{\kappa_{\beta_{i}}+i}, e_{\kappa_{\beta_{i}+1}+i}, \ldots, e_{\kappa_{a}+i}\right], \quad i=2,3, \ldots
$$


In the example we obtain $\beta_{2}=\beta_{3}=5$ and $\beta_{4}=\beta_{5}=\cdots=6$ and

$$
\begin{aligned}
& \mathcal{W}_{3}^{P}=\mathcal{W}_{2}^{P} \oplus \operatorname{im}\left[e_{9}\right] \\
& \mathcal{W}_{4}^{P}=\mathcal{W}_{3}^{P} \oplus \operatorname{im}\left[e_{10}\right] \\
& \mathcal{W}_{5}^{P}=\mathcal{W}_{4}^{P}, \quad \mathcal{W}_{6}^{P}=\mathcal{W}_{5}^{P}, \ldots
\end{aligned}
$$

Hence $\Delta_{i-1}=\operatorname{dim} \mathcal{W}_{i+1}-\operatorname{dim} \mathcal{W}_{i}=a-\left(\beta_{i}-1\right)$ and by induction $\Delta_{i}=a-\left(\beta_{i+1}-1\right)$. By definition, $\varepsilon_{\beta_{i-1}}=\cdots=\varepsilon_{\beta_{i}-1}=i$ and, therefore, $\varepsilon_{a-\Delta_{i-1}}=\cdots=\varepsilon_{a-\left(\Delta_{i}-1\right)}=$ $i$.

5.4. Proof of Theorem 3.2: Characterization of the solutions of associated DAE. The claim is a simple consequence of Lemmas 4.17 and 4.19 together with the well-known solution properties of a DAE corresponding to a regular DAE in (quasi-) Weierstraß form (see, e.g., [9]).

6. Conclusions. We have studied singular matrix pencils $s E-A$ and the associated DAE $E \dot{x}=A x+f$. With the help of the Wong sequences we were able to transform the matrix pencil into a quasi-Kronecker form (QKF). The QKF decouples the original matrix pencil into three parts: the underdetermined part, the regular part, and the overdetermined part. These blocks correspond to different solution behavior: existence but nonuniqueness (underdetermined part), existence and uniqueness (regular part), and possible nonexistence but uniqueness (overdetermined part). Furthermore, we have shown that the minimal indices of the pencil can be determined with only the Wong sequences.

7. Acknowledgments. We thank the anonymous reviewers for very helpful comments which helped us to improve this paper significantly. In particular, we added Theorem 2.9 in response to a question of one reviewer about the relationship between the Wong sequences and the minimal indices.

\section{REFERENCES}

[1] J. D. Aplevich, Minimal representations of implicit linear systems, Automatica J. IFAC, 21 (1985), pp. 259-269.

[2] J. D. Aplevich, Implicit Linear Systems, Lecture Notes in Control and Inform. Sci. 152, Springer-Verlag, Berlin, 1991.

[3] V. A. Armentano, The pencil $(s E-A)$ and controllability-observability for generalized linear systems: A geometric approach, SIAM J. Control Optim., 24 (1986), pp. 616-638.

[4] A. Banaszuk, M. KociȨCKI, AND F. L. Lewis, Kalman decomposition for implicit linear systems, IEEE Trans. Automat. Control, 37 (1992), pp. 1509-1514.

[5] A. Banaszuk, M. KociģCKi, and K. M. PrzyŁuski, Implicit linear discrete-time systems, Math. Control Signals Systems, 3 (1990), pp. 271-297.

[6] A. Banaszuk, M. KociȨCKI, And K. M. PrzyŁuski, Kalman-type decompostion for implicit linear discrete-time systems, and its applications, Internat. J. Control, 52 (1990), pp. 12631271.

[7] G. Basile and G. Marro, Controlled and Conditioned Invariants in Linear System Theory, Prentice-Hall, Englewood Cliffs, NJ, 1992.

[8] T. G. J. Beelen and P. Van Dooren, An improved algorithm for the computation of Kronecker's canonical form of a singular pencil, Linear Algebra Appl., 105 (1988), pp. 9-65.

[9] T. Berger, A. Ilchmann, and S. Trenn, The quasi-Weierstraß form for regular matrix pencils, Linear Algebra Appl., 436 (2012), pp. 4052-4069.

[10] P. Bernhard, On singular implicit linear dynamical systems, SIAM J. Control Optim., 20 (1982), pp. 612-633. 
[11] K.- W. E. Chu, The solution of the matrix equations $A X B-C X D=E$ and $(Y A-D Z, Y C-$ $B Z)=(E, F)$, Linear Algebra Appl., 93 (1987), pp. 93-105.

[12] J. W. Demmel And B. KÅGström, The generalized Schur decomposition of an arbitrary pencil $A-\lambda B:$ Robust software with error bounds and applications. Part I: Theory and Algorithms, ACM Trans. Math. Software, 19 (1993), pp. 160-174.

[13] J. W. Demmel And B. KÅgström, The generalized Schur decomposition of an arbitrary pencil $A-\lambda B$ : Robust software with error bounds and applications. Part II: Software and Applications, ACM Trans. Math. Software, 19 (1993), pp. 175-201.

[14] J. Dieudonné, Sur la réduction canonique des couples des matrices, Bull. Soc. Math. France, 74 (1946), pp. 130-146.

[15] H. Eliopoulou And N. Karcanias, Properties of reachability and almost reachability subspaces of implicit systems: The extension problem, Kybernetika, 31 (1995), pp. 530-540.

[16] F. R. Gantmacher, The Theory of Matrices (Vols. I 83 II), Chelsea, New York, 1959.

[17] V. Hernández and M. Gassó, Explicit solution of the matrix equation $A X B-C X D=E$, Linear Algebra Appl., 121 (1989), pp. 333-344.

[18] S. Iwata AND M. TAKAMATSU, On the Kronecker canonical form of mixed matrix pencils, SIAM J. Matrix. Anal. Appl., 32 (2011), pp. 44-71.

[19] L. Kronecker, Algebraische Reduction der Schaaren bilinearer Formen, Sitzungsber. Akad. Berlin, (1890), pp. 1225-1237.

[20] M. KuiJPer, First-Order Representations of Linear Systems, Birkhäuser Boston, Boston, 1994.

[21] P. Kunkel and V. Mehrmann, Differential-Algebraic Equations. Analysis and Numerical Solution, EMS Publishing House, Zürich, Switzerland, 2006.

[22] F. L. Lewis, A tutorial on the geometric analysis of linear time-invariant implicit systems, Automatica J. IFAC, 28 (1992), pp. 119-137.

[23] J. J. LoISEAU, Some geometric considerations about the Kronecker normal form, Internat. J. Control, 42 (1985), pp. 1411-1431.

[24] J. J. Loiseau, K. ÖzÇaldiran, M. Malabre, and N. Karcanias, Feedback canonical forms of singular systems, Kybernetika, 27 (1991), pp. 289-305.

[25] M. Malabre, Generalized linear systems: Geometric and structural approaches, Linear Algebra Appl., 122/123/124 (1989), pp. 591-621.

[26] C. OARĂ AND P. M. VAN DOOREN, An improved algorithm for the computation of structural invariants of a system pencil and related geometric aspects, Systems Control Lett., 30 (1997), pp. 39-48.

[27] D. H. Owens And D. L. DebeljKovic, Consistency and Liapunov stability of linear descriptor systems: A geometric analysis, IMA J. Math. Control \& Information, 2 (1985), pp. 139151.

[28] K. ÖZÇALdiRAn AND L. HALILOČLu, Structural properties of singular systems, Kybernetika, 29 (1993), pp. 518-546.

[29] K. M. PRZYŁuski AND A. M. Sosnowski, Remarks on the theory of implicit linear continuoustime systems, Kybernetika, 30 (1994), pp. 507-515.

[30] A. Sandovici, H. DE SNoo, And H. WinkleR, The structure of linear relations in Euclidean spaces, Linear Algebra Appl., 397 (2005), pp. 141-169.

[31] L. Schwartz, Théorie des Distributions à valeurs vectorielles, I and II, Hermann, Paris, 1957, 1958.

[32] H. J. S. Sмiтh, On systems of linear indeterminate equations and congruences, Philos. Trans. Roy. Soc. London, 151 (1861), pp. 293-326.

[33] S. Trenn, Distributional Differential Algebraic Equations, Ph.D. thesis, Institut für Mathematik, Technische Universität Ilmenau, Universitätsverlag Ilmenau, Ilmenau, Germany, 2009.

[34] S. Trenn, Regularity of distributional differential algebraic equations, Math. Control Signals Systems, 21 (2009), pp. 229-264.

[35] A. J. VAn Der Schaft And J. M. Schumacher, The complementary-slackness class of hybrid systems, Math. Control Signals Systems, 9 (1996), pp. 266-301.

[36] P. VAn Dooren, The computation of Kronecker's canonical form of a singular pencil, Linear Algebra Appl., 27 (1979), pp. 103-140.

[37] P. M. Van Dooren, Reducing subspaces: Definitions, properties, and algorithms, in Matrix Pencils, Lecture Notes in Math. 973, B. Kågström and A. Ruhe, eds., Springer-Verlag, Berlin, 1983, pp. 58-73.

[38] A. VARGA, Computation of Kronecker-like forms of a system pencil: applications, algorithms and software, in Proceedings of the IEEE International Symposium on Computer-Aided 
Control System Design, Dearborn, MI, 1996, pp. 77-82.

[39] J. H. Wilkinson, Linear differential equations and Kronecker's canonical form, in Recent Advances in Numerical Analysis, Carl de Boor and Gene H. Golub, eds., Academic Press, New York, London, 1978, pp. 231-265.

[40] K.-T. Wong, The eigenvalue problem $\lambda T x+S x$, J. Differential Equations, 16 (1974), pp. 270280. 